\title{
Estimating Mental Health Conditions of Patients with Opioid Use Disorder
}

\author{
Christopher Minnerly, ${ }^{1,2}$ Steven L. Bressler, ${ }^{3,4}$ Ibrahim M. Shokry, ${ }^{1,5}$ and Rui Tao $\mathbb{D}^{1}$ \\ ${ }^{1}$ Charles E. Schmidt College of Medicine, Florida Atlantic University, Boca Raton, FL, USA \\ ${ }^{2}$ FHE Health, Deerfield Beach, FL, USA \\ ${ }^{3}$ Center for Complex Systems and Brain Sciences, Florida Atlantic University, Boca Raton, FL, USA \\ ${ }^{4}$ Department of Psychology, Florida Atlantic University, Boca Raton, FL, USA \\ ${ }^{5}$ Ross University School of Veterinary Medicine, Basseterre, Saint Kitts and Nevis \\ Correspondence should be addressed to Rui Tao; rtao@health.fau.edu
}

Received 30 March 2019; Revised 14 July 2019; Accepted 21 August 2019; Published 26 September 2019

Academic Editor: Rebecca J. Houston

Copyright ( 2019 Christopher Minnerly et al. This is an open access article distributed under the Creative Commons Attribution License, which permits unrestricted use, distribution, and reproduction in any medium, provided the original work is properly cited.

\begin{abstract}
Objectives. Noninvasive estimation of cortical activity aberrance may be a challenge but gives valuable clues of mental health in patients. The goal of the present study was to characterize specificity of electroencephalogram (EEG) electrodes used to assess spectral powers associated with mental health conditions of patients with opioid use disorder. Methods. This retrospective study included 16 patients who had been diagnosed with opioid use disorder in comparison with 16 sex- and age-matched healthy controls. EEG electrodes were placed in the frontal (FP1, FP2, F3, F4, F7, F8, and Fz), central (C3, C4, and Cz), temporal (T3, T4, T5, and T6), parietal (P3, $\mathrm{P} 4$, and $\mathrm{Pz}$ ), and occipital scalp (O1 and $\mathrm{O} 2)$. Spectral powers of $\delta, \theta, \alpha, \beta$, and $\gamma$ oscillations were determined, and their distribution was topographically mapped with those electrodes on the scalp. Results. Compared to healthy controls, the spectral powers at low frequencies $(<8 \mathrm{~Hz} ; \delta$ and $\theta)$ were increased in most electrodes across the scalp, while powers at the high frequencies $(>12 \mathrm{~Hz} ; \beta$ and $\gamma)$ were selectively increased only at electrodes located in the frontal and central scalp. Among 19 electrodes, $\mathrm{F} 3, \mathrm{~F} 4, \mathrm{Fz}$, and $\mathrm{Cz}$ were highly specific in detecting increases in $\delta, \theta, \beta$, and $\gamma$ powers of patients with opioid use disorders. Conclusion. Results of the present study demonstrate that spectral powers are topographically distributed across the scalp, which can be quantitatively characterized. Electrodes located at F3, F4, Fz, and Cz could be specifically utilized to assess mental health in patients with opioid use disorders. Mechanisms responsible for neuroplasticity involving cortical pyramidal neurons and $\mu$-opioid receptor regulations are discussed within the context of changes in EEG microstates.
\end{abstract}

\section{Introduction}

The opioid epidemic in the US has hit an all-time high in recent years, with rates of affliction exponentially increasing. In 2016, an estimated 12 million people have used opioids for a variety of purposes, and approximately 2.1 million of those individuals suffer from mental disorders due to opioid uses [1]. The rates of opioid use disorder are projected to become worse in the next few years [2].

Opioid use disorder is often diagnosed with psychiatric evaluation based on the fifth edition of the Diagnostic and Statistical Manual of Mental Disorders (DMS-5; [3]). How brain activity is changed due to opioid use can only be determined with brain imaging techniques such as positron emission tomography (PET; [4]). However, high operating cost restricts the use of PET scans for the clinical evaluation. Electroencephalogram (EEG), which is commonly used to estimate neuronal disorders in patients with epilepsy or schizophrenia $[5,6]$, could be a more affordable alternative in estimating brain activity aberrance. EEG signals often reflect spatial and temporal activities of underlying cortical microcircuits consisting of pyramidal glutamatergic neurons, GABAergic interneurons, and subcortical inputs ([7]; also see a review by Cohen [8]). Opioids such as morphine, oxycodone, and heroin, exert their neurological effects 
mainly through activating $\mu$-opioid receptors that reside almost exclusively on GABAergic neurons $[9,10]$. The $\mu$-opioid receptors are functionally coupled with $\mathrm{G}$ proteingated inwardly rectifying $\mathrm{K}^{+}$(GIRK) channels, and activation of which results in hyperpolarization of GABAergic neurons and decreases in amplitude of inhibitory postsynaptic potentials (IPSPs) [11, 12]. Thus, opioids are considered as central nervous system (CNS) depressants, along with alcohol and benzodiazepines in substance abuse. EEG activity is increased when GABAergic neurons in the cortical microcircuits are disinhibited with opioids [13]. These microcircuits also receive afferent innervations from neurons of deep brain nuclei, which are regulated by opioids on GABAergic neurons [14]. Therefore, in general, there is a reduction in EEG activity following acute opioid administration as being demonstrated with drug-naïve humans $[15,16]$ and rodents as well [17]. However, longterm opioid administration causes an impairment of GIRK channels and GABAergic neurons [18, 19]. Furthermore, GABAergic hyperfunction is associated with drug dependence [20]. As a result, opioid use disordered individuals are more likely to have mental health conditions [21].

EEG is the summation of electrical signals that are measured as $\delta, \theta, \alpha, \beta$, and $\gamma$ oscillations [22]. This may raise the question as to what oscillations or rhythms are altered in patients with opioid use disorders. At which scalp electrodes are those changes being detected? Currently, is no consensus upon clinical relationship of EEG electrodes and rhythms altered in opioid patients (see reviews $[23,24])$. For instance, Wang et al. reported equal increases of $\delta, \theta, \alpha$, and $\beta$ powers in almost all EEG electrodes placed on the scalp [25]. Motlagh showed that $\beta$ but not $\delta, \theta$, or $\alpha$ oscillations were increased in opioid patients [26]. Although causes of inconsistency between reports are unknown, it suggests that it is necessary and also needed to re-evaluate the relationship of EEG electrodes and rhythms altered in opioid patients. Most recently, EEG analytical methods to investigate effects of drug abuse on $\delta, \theta, \alpha, \beta$, and $\gamma$ oscillations have been successfully developed in our laboratory $[27,28]$, which make it possible for us to reliably analyze rhythmic data extracted from patients.

We hypothesize that changes in EEG rhythms are characteristic of patients with opioid use disorder; furthermore, such changes occur at specific electrodes, which can be used to assess mental health conditions. Therefore, the aim of the present study was to characterize spectral rhythms recorded at EEG electrodes of patients with opioid use disorder compared to healthy controls. To fulfill this goal, we were first to reveal the normalized distribution of $\delta, \theta, \alpha, \beta$, and $\gamma$ rhythm powers using data from healthy controls. The subsequent analysis was to identify individual electrodes which showed significant changes in $\delta, \theta, \alpha, \beta$, or $\gamma$ powers of patients with opioid use disorders compared to those in healthy controls. Lastly, we examined whether spectral power distribution across the scalp was altered in patients with opioid use disorder.

\section{Materials and Methods}

2.1. Study Design. Data were obtained from an electronic medical database at a substance abuse treatment facility (FHE Health, Deerfield Beach, FL, USA), which had gathered $\sim 1000$ cases of information about patients' drug use history, DMS-5 diagnosis, and drug intoxication treatment. In addition, there were 20 cases obtained from healthy subjects with records indicating no substance abuse history. EEG data can be tracked electronically, along with information about detox-related symptoms. Searching with opioid-related keywords (i.e., morphine, heroin, fentanyl, methadone, or oxycodone), we found 350 patients who had records of opioid use history. However, a majority of patients were polysubstance users. After exclusion of alcohol, cocaine, and methamphetamine, only 100 subjects were considered as opioid users. Those with neurodegenerative diseases (e.g., Parkinson's and Alzheimer's), incomplete medical records, or low quality of EEG data were also excluded from the study. To this end, eleven men and five women identified with opioid use disorder were compared with 16 sex- and age-matched healthy controls. As shown in Table 1, patients had at least a three-year history of opioid abuse (average of 7.8 years). As a standard detox procedure, detoxification medications (buprenorphine) at a dosage of $8 \mathrm{mg}$ were given at the time of EEG recordings while abstinent from other opioid use for no more than one week (average of 2.4 days). No medication was given to sixteen healthy controls at EEG recording. The protocols of retrospective analysis of living subjects were approved by the Institutional Review Board (IRB) from Florida Atlantic University (Boca Raton, FL, USA) and Ross University School of Veterinary Medicine.

2.2. EEG Data Acquisition. In patients' medical records, EEG recording was performed between 12:00 PM and 4:00 PM. Following instrumental calibration, a case (patient or healthy control) was seated in a comfortable chair in a dimmed recording room, and the EEG procedures were orally instructed. A cap with 19 electrodes (Electro-Cap International, Eaton, OH, USA) was placed on the scalp. To reduce muscle artifacts in the EEG signal, the subject was instructed to assume a comfortable position and to avoid movement. Signals were collected with the band-pass filter of $1-100 \mathrm{~Hz}$ at a rate of $256 \mathrm{~Hz}$ and amplified with Neurofield's Q20 amplifier (NeuroField Inc., Bishop, CA, USA) using NeuroGuide software (Applied Neuroscience Inc., Tampa, FL, USA). Each subject underwent 10 minutes of EEG recording with eyes closed.

2.3. EEG Data Analysis. EEG data were downloaded from the database as described previously. Raw data were edited using the editing tool within the NeuroGuide software to remove physical artifacts (including eye movement, jaw movement, and gross movement). Test-retest reliability was used to govern EEG data quality after removal of the aforementioned artifacts. Theoretically, about $95 \%$ of the maximum change in EEG rhythms is reached within 
TABLE 1: Breakdown medical information of patients with opioid use disorder.

\begin{tabular}{|c|c|c|c|c|c|c|}
\hline Subject ID & Age (year) & Sex & Ethnicity $^{\mathrm{a}}$ & Drug $^{\mathrm{b}}$ & Years on drug & Other health issues \\
\hline $\mathrm{O} 2$ & 33 & M & $\mathrm{W}$ & $\mathrm{O}, \mathrm{H}$ & 14 & Bipolar disorder, unspecified \\
\hline $\mathrm{O} 3$ & 29 & M & $\mathrm{W}$ & $\mathrm{H}$ & 6 & N/A \\
\hline O6 & 31 & $\mathrm{~F}$ & $\mathrm{~W}$ & $\mathrm{H}$ & 5 & N/A \\
\hline O7 & 44 & M & $\mathrm{W}$ & $\mathrm{H}$ & 20 & Hepatitis C \\
\hline O8 & 22 & $\mathrm{~F}$ & $\mathrm{~W}$ & $\mathrm{H}$ & 4 & Hepatitis C \\
\hline $\mathrm{O} 13$ & 24 & M & $\mathrm{Hi}$ & $\mathrm{H}$ & 5 & Hepatitis C \\
\hline O15 & 51 & $\mathrm{M}$ & $\mathrm{W}$ & $\mathrm{O}$ & 4 & Hypertension \\
\hline $\mathrm{O} 17$ & 56 & $\mathrm{M}$ & $\mathrm{Hi}$ & $\mathrm{O}$ & 5 & Intermittent asthma \\
\hline O18 & 49 & M & $\mathrm{W}$ & $\mathrm{O}$ & 3 & Type II diabetes; hypertension \\
\hline $\mathrm{O} 26$ & 35 & M & $\mathrm{W}$ & $\mathrm{O}, \mathrm{H}$ & 15 & Psoriasis vulgaris \\
\hline $\mathrm{O} 28$ & 45 & $\mathrm{~F}$ & $\mathrm{~W}$ & $\mathrm{H}$ & 3 & Attention-deficit hyperactivity disorder \\
\hline O30 & 40 & M & $\mathrm{W}$ & $\mathrm{O}$ & 12 & N/A \\
\hline O31 & 29 & M & $\mathrm{W}$ & $\mathrm{H}$ & 7 & $\mathrm{~N} / \mathrm{A}$ \\
\hline $\mathrm{O} 33$ & 25 & $\mathrm{~F}$ & $\mathrm{~W}$ & $\mathrm{H}$ & 4 & N/A \\
\hline $\mathrm{O} 34$ & 49 & M & $\mathrm{Hi}$ & $\mathrm{O}$ & 13 & Gastroesophageal reflux disease; hypertension \\
\hline $\mathrm{O} 35$ & 22 & $\mathrm{~F}$ & W & $\mathrm{H}$ & 4 & $\mathrm{~N} / \mathrm{A}$ \\
\hline
\end{tabular}

${ }^{\mathrm{a}} \mathrm{W}$ denotes White; Hi, Hispanic. ${ }^{\mathrm{b}} \mathrm{M}$ denotes morphine; H, heroin; O, oxycodone.

minutes of recording time, and longer EEG time is not necessary to capture the bulk of the variability in EEG spectra. To estimate reliability, EEG spectral powers at a selected segment or epoch (i.e., $20 \mathrm{sec}, 40 \mathrm{sec}$, or $60 \mathrm{sec}$ ) are compared to those obtained from the whole segment (e.g., $10 \mathrm{~min}$ epoch). Reliability $(R)$ is equal to 0 if there is no regression of spectral powers between two epochs, or 1 if the same results are achieved from two epochs. It has been shown that $20 \mathrm{sec}$ epochs are typically to have 0.8 reliable, 40 -sec epochs about 0.9 reliable, and 60 -sec epochs at 0.95 reliable [29]. In the present study, we decided to use 60-sec epoch with reliable levels at 0.90 or greater. Nevertheless, a 60 -sec epoch randomly selected from the artifact-free EEG graph $(R \geq 0.9)$ was subjected to EEG spectral power analysis using a fast Fourier transform (FFT), by which graphic images were mathematically transformed into voltage powers showing on $y$-axis $\left(\mu \mathrm{V}^{2} / \mathrm{Hz}\right)$ plotted against spectral frequencies on $x$-axis $(1-50 \mathrm{~Hz} ; \delta, 1-4 \mathrm{~Hz} ; \theta, 4-$ $8 \mathrm{~Hz} ; \alpha, 8-12 \mathrm{~Hz} ; \beta, 12-30 \mathrm{~Hz}$; and $\gamma, 30-50 \mathrm{~Hz})$. The transformed numbers including both spectral powers $\left(\mu \mathrm{V}^{2} /\right.$ $\mathrm{Hz}$ ) and frequencies $(\mathrm{Hz})$ were then copy-pasted to a Microsoft Excel sheet for further data calculation. Powers of $\delta, \theta, \alpha, \beta$, or $\gamma$ oscillations were individually sorted according to electrodes and averaged (mean \pm SEM). The relationships between 5 spectral powers and 19 electrodes were determined in three distinct ways. First, the normal distribution of spectral powers $(\delta, \theta, \alpha, \beta$, and $\gamma)$ across different parts of the cortex was characterized. Specifically in healthy controls, data obtained from different areas were compared, including the frontal versus rear components, the left versus right components, and further dissected into the frontal, temporal, central, parietal, and occipital components. With such groundwork, we revealed differences in power levels between brain areas or lobes. Results of this analysis are presented in Tables 2-4. Next, spectral powers of $\delta, \theta, \alpha, \beta$, or $\gamma$ oscillations at individual electrodes in patients with opioid use disorder were compared with those of healthy controls, across all electrode sites (frontal: FP1, FP2, F3, F4, Fz, F7, and F8; central: C3, Cz, and C4;
TABle 2: Comparative analysis of power levels distributed on the scalp of healthy controls. Data were based on 16 healthy controls.

\begin{tabular}{lcccc}
\hline $\begin{array}{c}\text { Frontal scalp } \\
\left(F ; \mu \mathrm{V}^{2}\right)^{\mathrm{b}}\end{array}$ & Rear scalp $\left(R ; \mu \mathrm{V}^{2}\right)$ & $F / R$ ratio & $P$ value $^{\mathrm{c}}$ \\
\hline$\delta^{\mathrm{a}}$ & $13.2 \pm 0.1$ & $10.4 \pm 0.1$ & 1.3 & $P<0.001$ \\
$\theta$ & $6.9 \pm 0.1$ & $8.8 \pm 0.1$ & 0.8 & $P>0.05$ \\
$\alpha$ & $12.4 \pm 0.1$ & $35.2 \pm 0.6$ & 0.4 & $P<0.001$ \\
$\beta$ & $6.4 \pm 0.1$ & $11.6 \pm 0.1$ & 0.6 & $P<0.001$ \\
$\gamma$ & $1.6 \pm 0.1$ & $1.7 \pm 0.1$ & 0.9 & $P>0.05$ \\
\hline
\end{tabular}

${ }^{\mathrm{a}} \delta, 1-4 \mathrm{~Hz} ; \theta, 4-8 \mathrm{~Hz} ; \alpha, 8-12 \mathrm{~Hz} ; \beta, 12-30 \mathrm{~Hz}$; and $\gamma, 30-50 \mathrm{~Hz} .{ }^{\mathrm{b}}$ Frontal (F), FP1, FP2, F3, F4, F7, F8, and Fz); Rear (R), O1, O2, P3, P4, T5, T6, and Pz). Note that the electrode located in the middle (T3, T4, C3, C4, and Cz) were excluded in the data analysis. 'Significance was determined by repeated measures ANOVA.

TABLe 3: Comparative analysis of power levels distributed on the scalp of healthy controls. Data were based on 16 healthy controls.

\begin{tabular}{ccccc}
\hline & Left scalp $\left(L ; \mu \mathrm{V}^{2}\right)$ & Right scalp $\left(R ; \mu \mathrm{V}^{2}\right)$ & $L / R$ ratio & $P$ value \\
\hline$\delta$ & $11.2 \pm 0.1$ & $11.1 \pm 0.1$ & 1.0 & $>0.05$ \\
$\theta$ & $7.4 \pm 0.1$ & $6.8 \pm 0.1$ & 1.1 & $>0.05$ \\
$\alpha$ & $20.9 \pm 0.3$ & $20.7 \pm 0.3$ & 1.0 & $>0.05$ \\
$\beta$ & $8.5 \pm 0.1$ & $8.6 \pm 0.1$ & 1.0 & $>0.05$ \\
$\gamma$ & $1.8 \pm 0.1$ & $1.8 \pm 0.1$ & 1.0 & $>0.05$ \\
\hline
\end{tabular}

Note that comparative analysis of power levels recorded in the left (FP1, F3, F7, C3, T3, F3, T5, and O1) versus right scalps (FP2, F4, F8, C4, T4, F4, T6, and O2).

The electrodes at midline $(\mathrm{Fz}, \mathrm{Cz}$, and $\mathrm{Pz})$ were excluded from the data analysis.

TABLE 4: Comparative analysis of power levels distributed on scalp of healthy controls. Data were based on 16 healthy controls.

\begin{tabular}{cccccc}
\hline & Frontal $^{\mathrm{a}}$ & Central $^{\mathrm{b}}$ & Temporal $^{\mathrm{c}}$ & Parietal $^{\mathrm{d}}$ & Occipital \\
\hline$\delta$ & $13.2 \pm 0.1$ & $12.3 \pm 0.1$ & $8.2 \pm 0.1^{\mathrm{a}, \mathrm{b}}$ & $11.6 \pm 0.1^{\mathrm{c}}$ & $10.0 \pm 0.1^{\mathrm{c}}$ \\
$\theta$ & $6.9 \pm 0.1$ & $8.8 \pm 0.1$ & $5.8 \pm 0.1^{\mathrm{b}}$ & $9.2 \pm 0.1^{\mathrm{c}}$ & $9.3 \pm 0.4^{\mathrm{c}}$ \\
$\alpha$ & $12.4 \pm 0.1$ & $20.7 \pm 0.6$ & $16.5 \pm 0.5$ & $33.1 \pm 1.1^{\mathrm{a}, \mathrm{b}, \mathrm{c}}$ & $48.1 \pm 2.8^{\mathrm{a}, \mathrm{b}, \mathrm{c}}$ \\
$\beta$ & $6.4 \pm 0.1$ & $9.6 \pm 0.2^{\mathrm{a}}$ & $7.7 \pm 0.1$ & $11.5 \pm 0.2^{\mathrm{a}, \mathrm{c}}$ & $13.9 \pm 0.5^{\mathrm{a}, \mathrm{b}, \mathrm{c}}$ \\
$\gamma$ & $1.6 \pm 0.1$ & $1.5 \pm 0.1$ & $2.1 \pm 0.1^{\mathrm{b}}$ & $1.4 \pm 0.1^{\mathrm{c}}$ & $2.4 \pm 0.1^{\mathrm{a}, \mathrm{b}, \mathrm{d}}$ \\
\hline
\end{tabular}

Note that electrodes placed on scalp of the frontal (FP1, FP2, F3, F4, F7, F8, and $\mathrm{Fz}$ ), temporal (T3, T4, T5, and $\mathrm{T} 6)$, central ( $\mathrm{C} 3, \mathrm{C} 4$, and $\mathrm{Cz})$, parietal (P3, $\mathrm{P} 4$, and $\mathrm{Pz}$ ) and occipital areas (O1 and $\mathrm{O} 2)$. Significance were determined by repeated measures ANOVA followed by post hoc Scheffe test. ${ }^{a} P<0.05$ vs. Frontal. ${ }^{\mathrm{b}} P<0.05$ vs. Temporal. ${ }^{\mathrm{c}} P<0.05$ vs. Central. ${ }^{\mathrm{d}} P<0.05$ vs. Parietal. 
temporal: T3, T4, T5, and T6; and parietal-occipital: $\mathrm{P} 3, \mathrm{Pz}$, $\mathrm{P} 4, \mathrm{O} 1$, and $\mathrm{O} 2$ ). To reveal topographic distribution of spectral powers in the brain, power levels were arbitrarily categorized into four groups as follows: high $>76 \%$; medium 51-75\%; low 11-50\%; no change $<10 \%$. After initial analysis, we found that for most cases the spectral powers of counterpart electrodes at two hemispheres were almost identical, and thus the counterpart electrodes were always grouped together. There were only a few cases that the power levels were different between two hemispheres; if it occurred, the counterpart electrodes were categorized into the low-level change group. Results of the analysis are presented in Figures $1-5$. The third analysis was to reveal what EEG electrodes could have more specific changes than others. To fulfill this aim, data were analyzed from several aspects, including analyses of spectral powers, brain lobes, or EEG electrodes. Furthermore, Venn diagram analysis was used to determine specificity of electrodes that could detect changes in spectral powers, including all 5 spectra (i.e., $\delta, \theta, \alpha, \beta$, and $\gamma$ oscillations). Results of this analysis are presented in Table 5 and Figures 6 and 7 .

2.4. Statistical Analysis. All data are expressed as mean \pm SEM and have been evaluated with repeated measures ANOVA between subjects (e.g., frontal vs. rear areas and patients vs healthy controls) followed by post hoc Scheffe test using StatView software 5.0 (SAS Institute Inc., Cary, NC, USA). Unpaired Student's $t$-test was also utilized to determine statistical differences if appropriated. Significance was set at 0.05.

\section{Results}

3.1. A Normal Distribution of Spectral Powers across the Scalp in Healthy Controls. First, we analyzed 16 healthy controls to determine whether spectral powers of $\delta, \theta, \alpha, \beta$, or $\gamma$ oscillations were different between the frontal (FP1, FP2, F3, F4, F7, F8, and Fz) versus rear scalps (O1, O2, P3, P4, T5, T6, and $\mathrm{Pz}$ ). As illustrated in Table $2, \delta$ powers were significantly higher in the frontal sites compared to the rear sites $\left(F_{(1,222)}=14.188, P=0.0002\right)$. There was no significant difference in $\theta$ powers between the two areas $\left(F_{(1,222)}=0.522\right.$, $P=0.4708)$. As the frequencies increased to $\alpha$ or $\beta$ oscillation, spectral powers were significantly lowered in the frontal sites relative to the rear sites $\left(\alpha, F_{(1,222)}=14.027\right.$, $\left.P=0.0002 ; \beta, F_{(1,222)}=22.143, P<0.0001\right)$. For the $\gamma$ oscillations, the powers at the frontal sites were almost the same as the rear sites, showing no statistical difference $\left(F_{(1,222)}=0.522, P=0.4708\right)$.

Next, spectral powers on the left hemisphere (FP1, F3, F7, C3, T3, F3, T5, and O1) were compared with those of the right (FP2, F4, F8, C4, T4, F4, T6, and O2). Data analysis revealed no difference in spectral powers between the two hemispheres (Table 3).

Lastly, electrodes were regrouped into the frontal (FP1, FP2, F3, F4, F7, F8, and Fz), central (C3, C4, and $\mathrm{Cz})$, temporal (T3, T4, T5, and T6), parietal (P3, P4, and $\mathrm{Pz}$ ), and occipital (O1 and $\mathrm{O} 2$ ) areas. Spectral powers were significantly different between those areas (Table 4). Further data analysis revealed that $\alpha$ and $\beta$ powers were low in the frontal areas and markedly increased in a range of $100 \%-$ $300 \%$ in the occipitals. In contrast, changes of $\delta, \theta$, and $\gamma$ powers were limited, with less than $50 \%$ difference between areas.

\subsection{Changes in Powers of Patients with Opioid Use Disorders}

3.2.1. Changes in $\delta$ Powers. Figure 1(a) displays samples of $10 \mathrm{~s} \delta$-oscillations obtained from a Cz electrode placed in the midline center on the scalp. It appears that the $\delta$ powers were increased in a patient with opioid use disorder (Figure 1(a) bottom) as compared with an age- and sex-matched healthy subject (Figure 1(a) top). Changes of $\delta$ powers across $19 \mathrm{EEG}$ electrodes were arbitrarily classified into 4 levels: high (>76\%), medium (51-75\%), low (11-50\%), and no change $(<10 \%)$. Figure $1(\mathrm{~b})$ shows 4 electrodes having more than $>76 \%$ increases in $\delta$ powers. The results of two-factor ANOVA indicated a significant main effect for opioid use $\left(F_{(1,30)}=7.874, P=0.0087\right)$ and a significant main effect for electrode locations $\left(F_{(18,540)}=10.475, P<0.00001\right)$. Specifically, all were located in the midline centers and occipitals, including $\mathrm{Cz}, \mathrm{Pz}, \mathrm{O} 1$, and $\mathrm{O} 2$. In the healthy controls, $\delta$ powers $\left(\mu \mathrm{V}^{2} ; N=16\right)$ were $13.5 \pm 0.3,11.7 \pm 0.3,10.2 \pm 0.3$, and $9.8 \pm 0.3$, respectively. The $\delta$ powers in those electrodes of opioid patients were increased by $80 \%, 101 \%, 86 \%$, and $84 \%$, respectively.

Figure 1(c) shows a medium increase $(51-75 \%)$ of $\delta$ powers in the frontal (F3 and F4), central (C3, Fz and $\mathrm{C} 4)$, parietal (P3 and $\mathrm{P} 4)$, and temporal areas (T3, T4, T5, and T6). The increase was significant $\left(F_{(1,30)}=8.726, P=0.0061\right)$.

Figure $1(\mathrm{~d})$ shows an $11-50 \%$ increase of $\delta$ powers in the FP1, FP2, F7, and F8 electrodes. However, the increased $\delta$ powers were not statistically different from those of the healthy controls $\left(F_{(1,30)}=2.115, P=0.156\right)$. Figure $1(\mathrm{e})$ displays topographical mapping of distribution of $\delta$ powers categorized with high, medium, and low levels. The red area denotes a high-activity increase of $\delta$ powers, the green for the medium-activity increase, and the brown for the low-activity increase of $\delta$ powers.

3.2.2. Changes in $\theta$ Powers. Figure 2(a) displays samples of $10 \mathrm{~s} \theta$ oscillations obtained from the FP1 electrode in a patient with opioid use disorder (Figure 2(a) bottom) as compared to a healthy control (Figure 2(b) top). Figure 2(b) shows the group of electrodes recorded with high $\theta$ power changes ( $>76 \%$ vs. healthy control). They were located in the frontal (FP1, FP2, F3, and F4), middle-central (Fz, C3, $\mathrm{Cz}$, and $\mathrm{C} 4$ ), and temporal areas (T3 and T4). Two-way repeated measures ANOVA revealed a significant main effect for opioid patients $\left(F_{(1,30)}=4.792, P=0.037\right)$ and a significant main effect for the 19-electrode placements $\left(F_{(18,540)}=13.135, P<0.0001\right)$. Except for the T3 electrode, post hoc Scheffe test revealed the increase was significantly different from the respective electrodes of the healthy controls. 


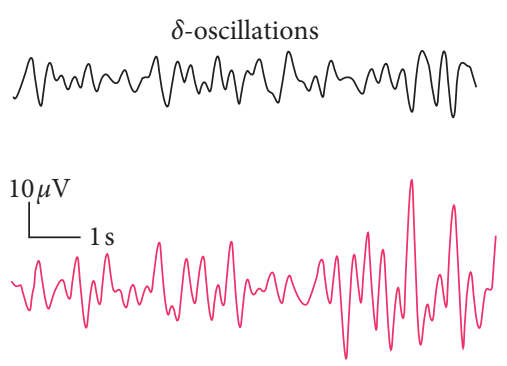

(a)

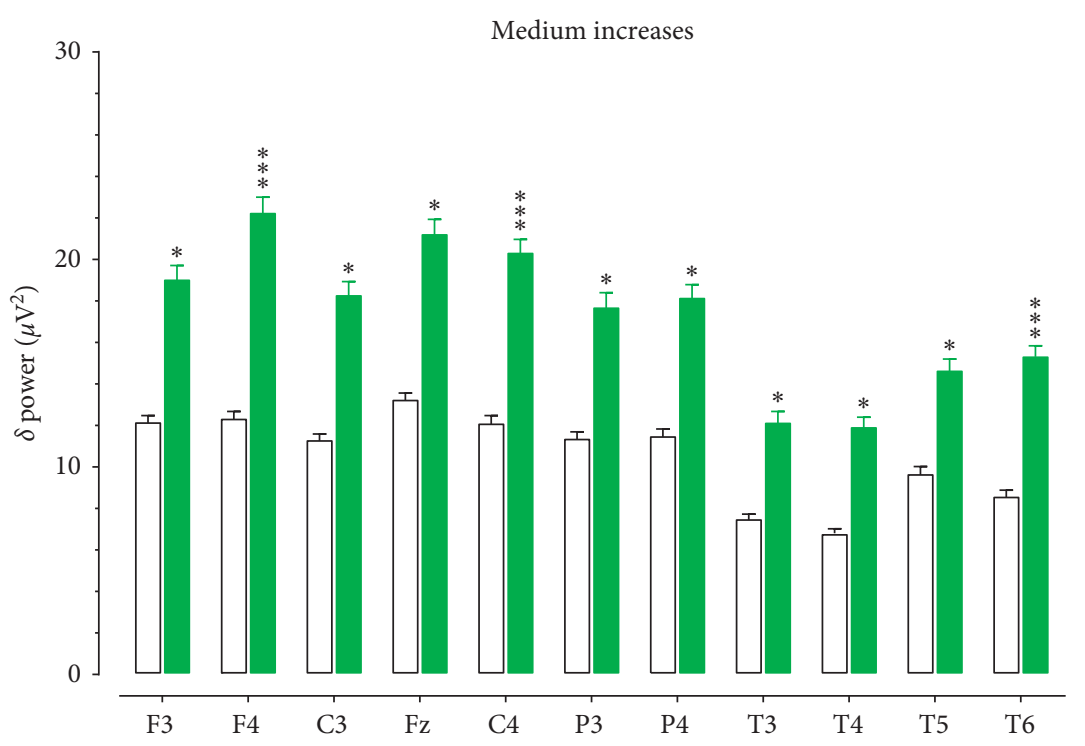

(c)

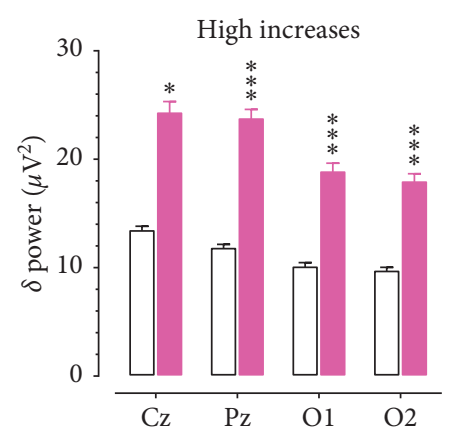

(b)

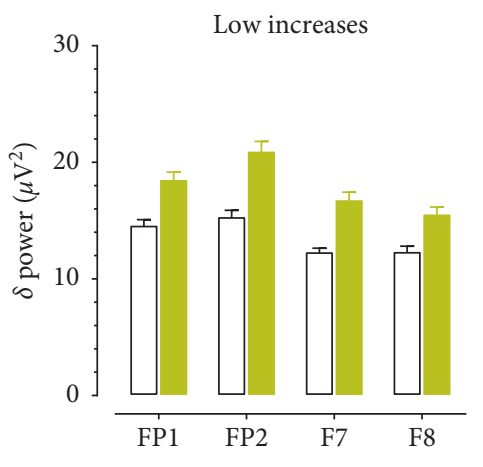

(d)

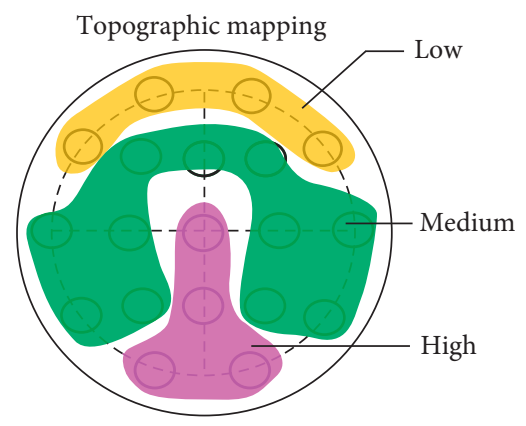

(e)

FIGURE 1: Topographic changes of $\delta$ powers in patients with opioid use disorder. (a) EEG traces of $\delta$ oscillations (1-4 Hz) representing $10 \mathrm{~s}$ activity from a healthy control (Top) and a patient with opioid use disorder. Horizontal scale bar, $1 \mathrm{~s}$; vertical scale bar, $10 \mu \mathrm{V}$. (b) The $\delta$ powers were increased by $>76 \%$ in the $\mathrm{Cz}, \mathrm{Pz}, \mathrm{O} 1$, and $\mathrm{O} 2$ electrodes. Open columns denote healthy controls and solid red columns indicate the opioid group. ${ }^{*} P<0.05$, ${ }^{* * *} P<0.001$ vs. healthy controls determined by repeated measures ANOVA followed by post hoc Scheffe test. (c) The $\delta$ powers were increased by $51-75 \%$ in F3, F4, C3, Fz, C4, P3, P4, T3, T4, T5, and T6. Open columns denote the healthy control group, and solid green columns indicate the opioid group. ${ }^{*} P<0.05,{ }^{* * *} P<0.001$ vs. healthy control determined by repeated measures ANOVA followed by post hoc Scheffe test. (d) The $\delta$ powers were increased by $11-50 \%$ in FP1, FP2, F7, and F8. Open columns denote the healthy control group, and solid brown columns indicate the opioid group. NS, $P>0.05$ vs. healthy control determined by repeated measures ANOVA. (e) Topographic correlation between increased $\theta$ oscillation (1-4 Hz) and electrodes on cortical scalps. The red area denotes high $\delta$ powers, the green for the medium $\delta$ powers, and the brown for the low $\delta$ powers.

Overall, a medium-activity increase (51-75\%; Figure 2(c)) of $\theta$ powers was found in 7 electrodes (Figure 3(c); F7, F8, P3, $\mathrm{P} 4, \mathrm{Pz}, \mathrm{O} 1$, and $\mathrm{O} 2)$. Statistical analysis revealed that $\theta$ changes at those 7 electrodes were not different from the control group $(P>0.05)$. A low increase $(11-50 \%$; Figure 2(d)) of $\theta$ powers was found in the T5 and T6 electrodes, and this effect was not significant $(P>0.05)$. Topographical changes of $\theta$ powers on the scalp are shown in 

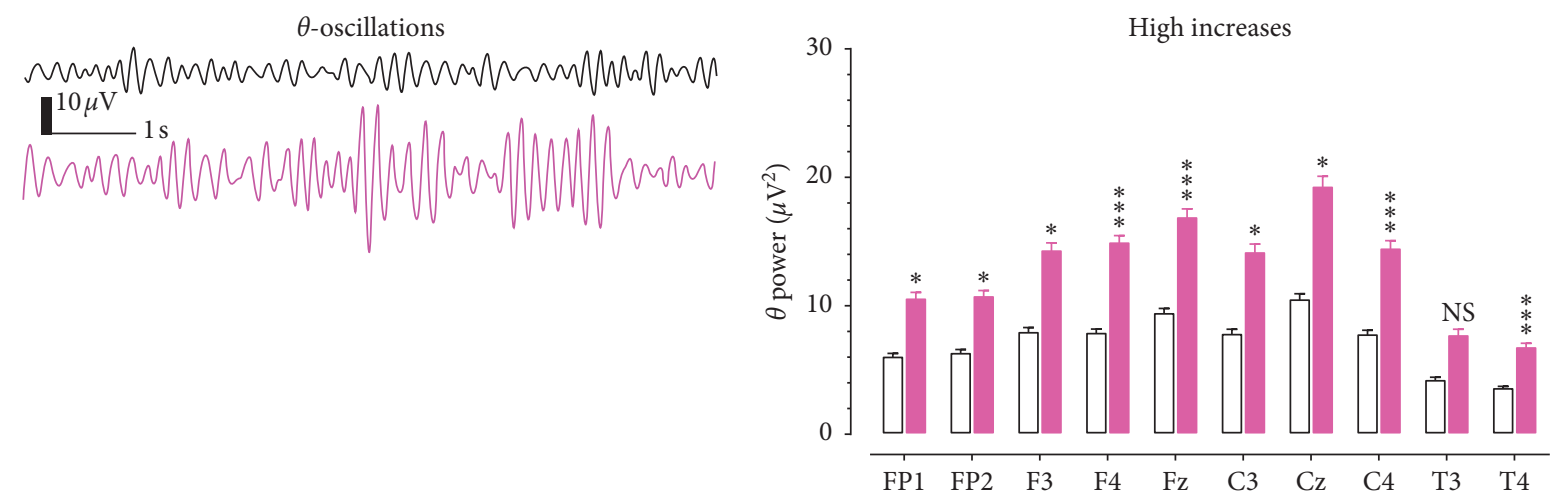

(a)

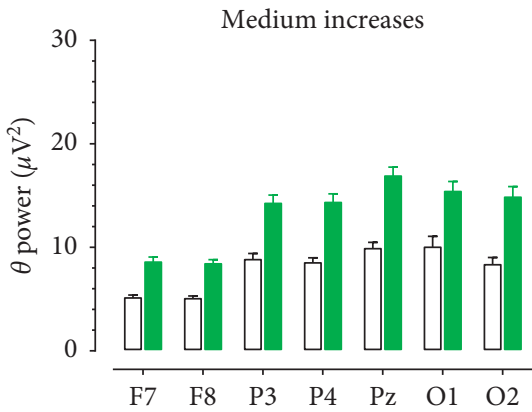

(c) (b)

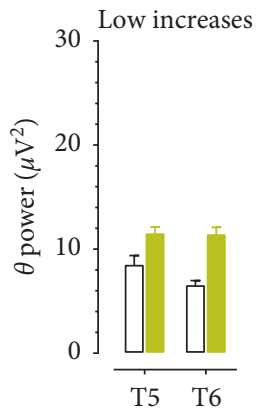

(d)

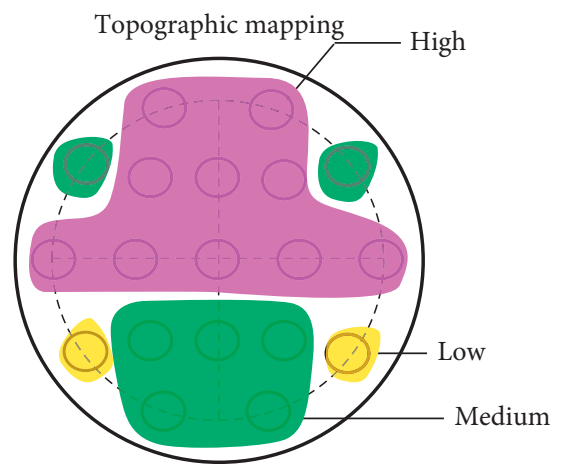

(e)

FIgURE 2: Topographic changes of $\theta$ powers in patients with opioid use disorder. (a) Examples of EEG traces representing $10 \mathrm{~s} \theta$ oscillations from a healthy control (top) and a patient with opioid use disorder. Horizontal scale bar, 1 s; vertical scale bar, $10 \mu \mathrm{V}$. (b) The $\theta$ powers was increased by $>76 \%$, including FP1, FP2, F3, F4, Fz, C3, Cz, C4, T3, and T4. Open columns denote healthy controls, and solid red columns indicate the opioid group. NS, $P>0.05,{ }^{*} P<0.05,{ }^{* * *} P<0.001$ vs. healthy controls determined by repeated measures ANOVA followed by post hoc Scheffe test. (c) The $\theta$ powers were increased by $51-75 \%$ in F7, F8, P3, P4, Pz, O1, and O2. Open columns denote the healthy control group, and solid green columns indicate the opioid group. NS, $P>0.05$ vs. healthy control determined by repeated measures ANOVA. (d) The $\theta$ powers were increased by $11-50 \%$ in T5 and T6. Open columns denote the healthy control group, and solid brown columns indicate a low change in the opioid group. NS, $P>0.05$ vs. healthy control determined by repeated measures ANOVA. (e) Topographic correlation between increased $\theta$ oscillation $(4-8 \mathrm{~Hz})$ and electrodes on cortical scalps. The red area denotes for high $\delta$ powers, the green for medium $\delta$ powers, and the brown for low $\delta$ powers.

Figure 2(e). The red area denotes a high increase of $\theta$ powers, the green for the medium increase, and the brown for the low increase of $\theta$ powers.

3.2.3. Changes in $\alpha$ Powers. Figure 3(a) displays samples of $10 \mathrm{~s} \alpha$ oscillations obtained from the $\mathrm{O} 1$ electrode in a patient in contrast to the healthy controls. There was a low reduction (10-50\%) of $\alpha$ oscillations in the $\mathrm{O} 1, \mathrm{O} 2$,
T5, T6, P3, P4, and Pz electrodes (Figure 3(b)). However, the reduction was not statistically different from that obtained from the healthy control $\left(F_{(1,30)}=0.368\right.$, $P=0.549$ ).

Changes in the remaining electrodes were less than $10 \%$, including FP1, FP2, F3, F4, F7, F8, Fz, T3, C3, Cz, C4, and T4 (Figure 3(c)). The dark area in Figure 3(d) represents topographical reduction of the $\alpha$ powers in the temporal, parietal, and occipital areas. 


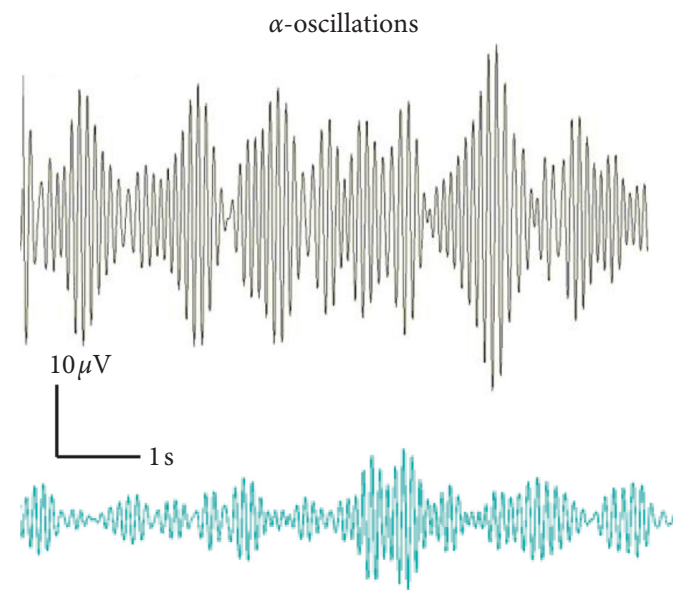

(a)

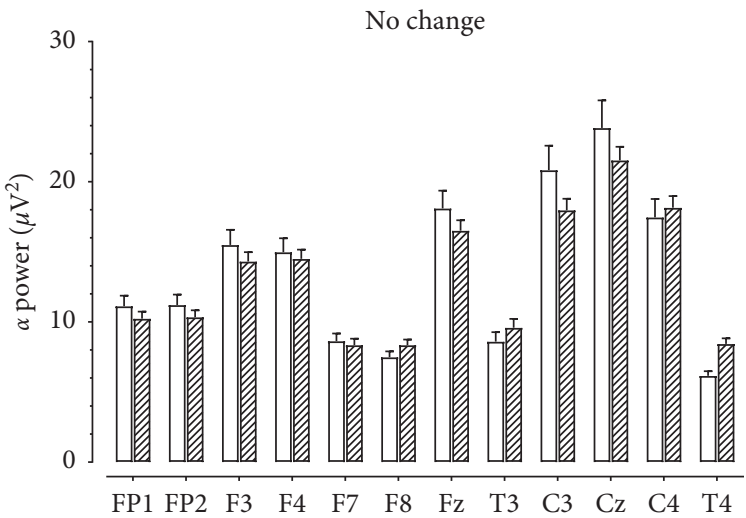

(c)

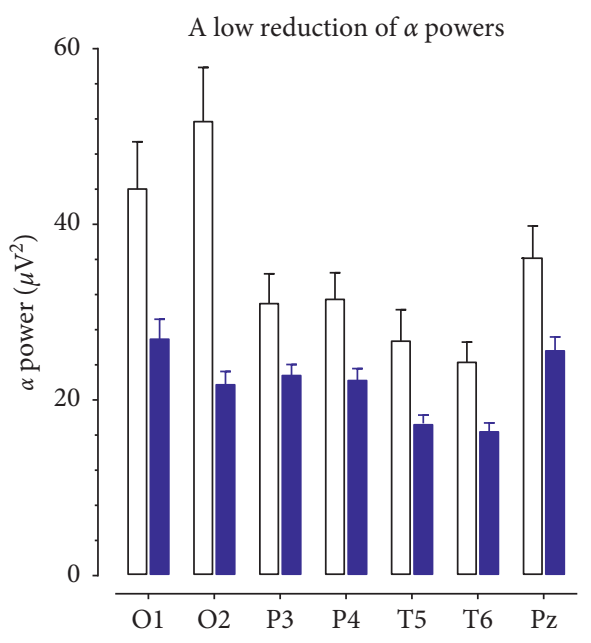

(b)

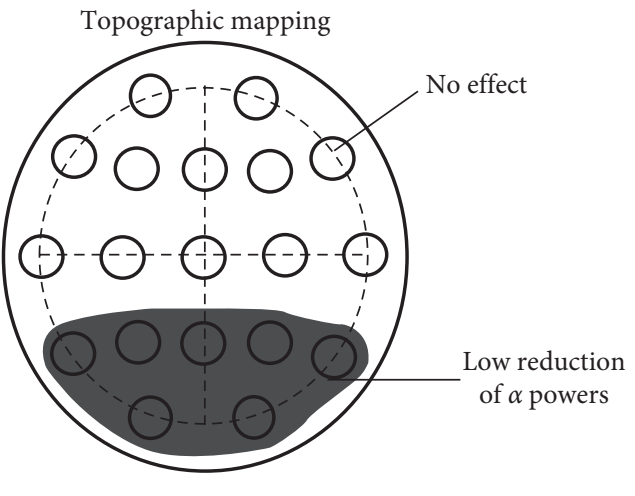

(d)

FIGURE 3: Topographic changes of $\alpha$ powers in patients with opioid use disorder. (a) Examples of EEG traces representing $10 \mathrm{~s} \alpha$ oscillations from a healthy control (top) and a patient with opioid use disorder. Horizontal scale bar, $1 \mathrm{~s}$; vertical scale bar, $10 \mu \mathrm{V}$. (b) The $\alpha$ powers was reduced by 11-50\% in O1, O2, P3, P4, T5, T6, and Pz. Open columns denote for the healthy control group and solid blue columns for a reduction of $\alpha$ oscillations in the opioid group. NS, $P>0.05$ vs. healthy controls determined by repeated measures ANOVA. (c) There was no change of $\alpha$ powers in FP1, FP2, F3, F4, F7, F8, Fz, T3, C3, Cz, C4, or T4. Open columns denote for the healthy control group, and zebrastriping columns for the opioid group. NS, $P>0.05$ vs. healthy control determined by repeated measures ANOVA. (d) Topographic correlation between reduced $\alpha$ oscillation $(8-12 \mathrm{~Hz})$ and electrodes on the scalp. The dark area denotes for a low reduction of $\alpha$ powers.

3.2.4. Changes in $\beta$ Powers. Figure 4(a) displays samples of $10 \mathrm{~s} \beta$ oscillations obtained from F3 electrodes. The $\beta$-amplitude was increased in a patient with opioid use disorder (Figure 5(a) bottom) as compared to a healthy control (Figure 4 (a) top). However, no activity exceeding $76 \%$ was found in any electrode. Figure $4(\mathrm{~b})$ shows the group of electrodes recorded with medium $\delta$ power changes (51$75 \%)$. Results of two-way repeated measures ANOVA show a significant main effect for opioid patients $\left(F_{(1,30)}=10.359\right.$, $P=0.031)$ and a significant main effect for the 19-lead electrode placements $\left(F_{(18,540)}=12.167, P<0.0001\right)$. A low increase (11-50\%) was found in the FP1, FP2, C3, C4, F7, and F8 electrodes (Figure 4(c)). The change had no statistically significant difference from the respective electrodes obtained from the healthy controls $\left(F_{(1,30)}=2.928\right.$, $P=0.097)$. Changes in the P3, P4, T3, T4, T5, T6, and Pz electrodes (Figure $4(\mathrm{~d})$ ) were less than $10 \%$ and not different from the controls $\left(F_{(1,30)}=0.091, P=0.766\right)$. Lastly, $\beta$ powers obtained from $\mathrm{O} 1$ and $\mathrm{O} 2$ electrodes were evaluated, finding a tendency of a low reduction in the occipitals (Figure 4(e)). However, the reduction was not statistically different from the healthy controls $\quad\left(F_{(1,30)}=0.238\right.$, $P=0.629$ ). Figure $4(\mathrm{f})$ displays $\beta$ power levels topographically distributed in the scalp. The green area denotes a medium increase of $\beta$ powers, the brown for the low increase, and the dark represents the low reduction of $\beta$ powers.

3.2.5. Changes in $\gamma$ Powers. Figure 5(a) displays samples of $10 \mathrm{~s} \gamma$-oscillations obtained from F3 electrodes, showing increases of $\gamma$-amplitudes in an opioid patient (bottom) in contrast to a healthy control (top). Figure 5(b) shows the group of electrodes recorded with a medium $\gamma$ power increase, including F3, F4, Fz, and $\mathrm{Cz}$ electrodes. The results of two-factor ANOVA indicated a significant main effect for opioid use $\left(F_{(1,30)}=54.084, P<0.001\right)$ and a significant main effect for electrode locations $\left(F_{(18,540)}=15.268, P<0.00001\right)$. 

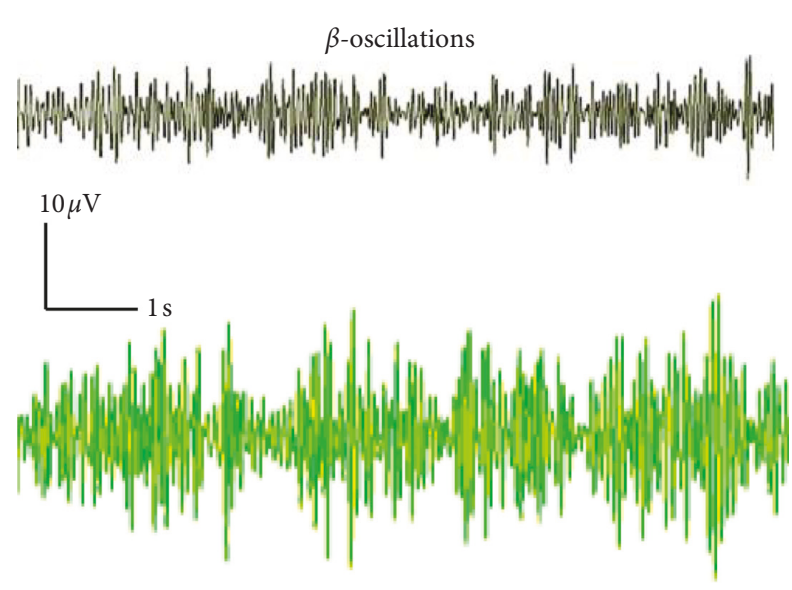

(a)

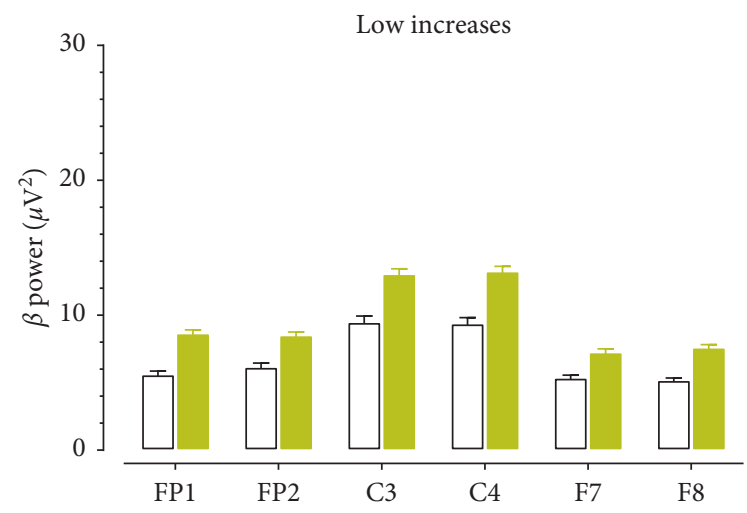

(c)

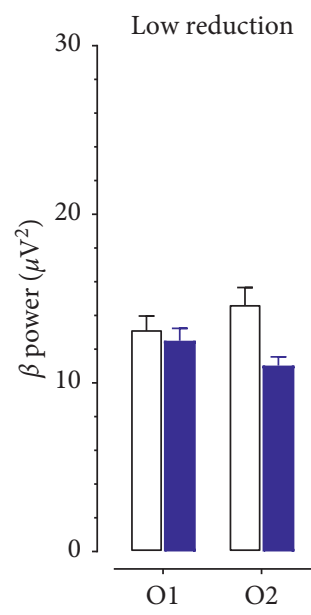

(e)

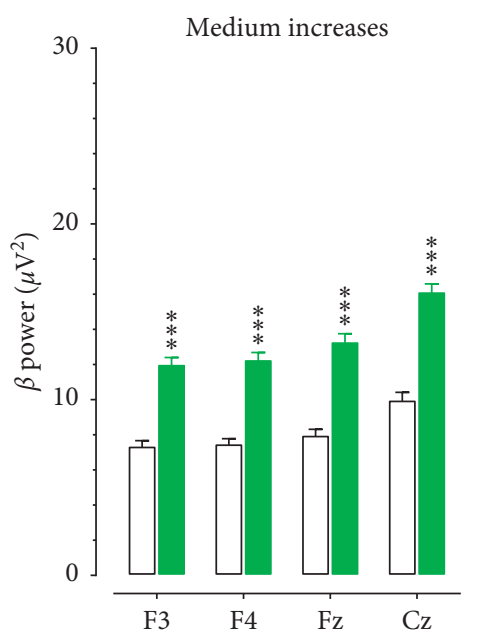

(b)

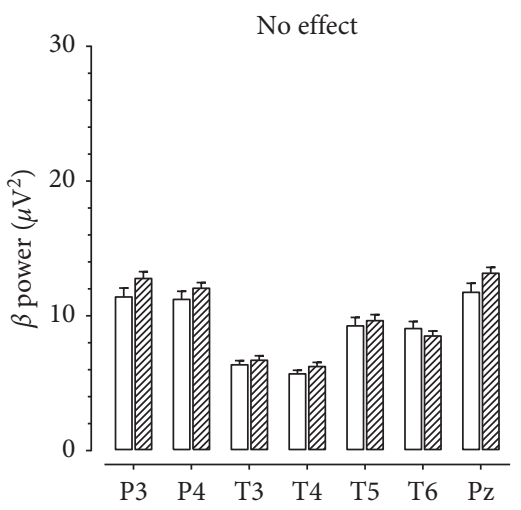

(d)

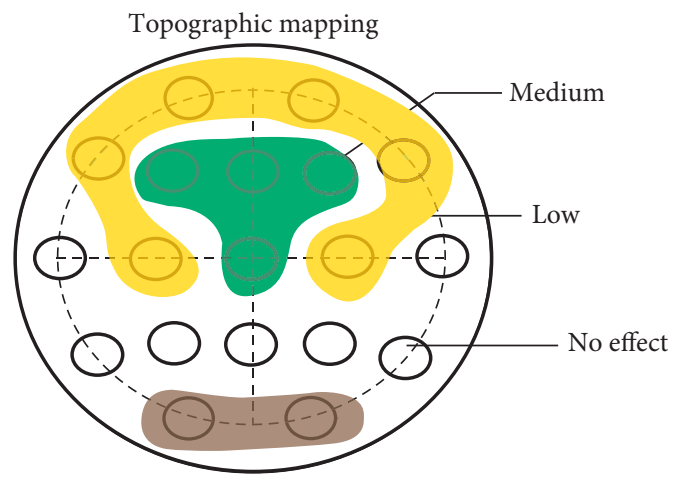

(f)

FIgURE 4: Topographic changes of $\beta$ powers in patients with opioid use disorder. (a) Examples of EEG traces representing $10 \mathrm{~s} \beta$ oscillations from a healthy control (top) and a patient with opioid use disorder. Horizontal scale bar, $1 \mathrm{~s}$; vertical scale bar, $10 \mu \mathrm{V}$. (b) The increase of $\beta$ powers was 51-75\%, including F3, F4, Fz, and Cz. Open columns denote for the healthy control group and solid green columns for the opioid group. NS, $P>0.05$. ${ }^{* * *} P<0.001$ vs. healthy controls determined by repeated measures ANOVA followed by post hoc Scheffe test. (c) The increase of $\beta$ powers was 11-50\% in FP1, FP2, C3, C4, F7, and F8. Open columns denote for the healthy control group and solid brown columns for a low change in the opioid group. NS, $P>0.05$ vs. healthy control determined by repeated measures ANOVA. (d) The increase of $\beta$ powers was $<10 \%$ in P3, P4, T3, T4, T5, T6, and Pz. Open columns indicate the healthy control group, and zebra-striping columns denote for "no change" in the opioid group. NS, $P>0.05$ vs. healthy control determined by repeated measures ANOVA. (e) A tendency of $\beta$ powers reduction was found in $\mathrm{O} 1$ and $\mathrm{O} 2$. Open columns denote for the healthy control group, and solid blue columns for a reduction of $\beta$ oscillations in the opioid group. NS, $P>0.05$ vs. healthy control determined by repeated measures ANOVA. (f) Topographic correlation between $\beta$ oscillation $(12-30 \mathrm{~Hz})$ and electrodes on the scalp. The green area denotes for the medium increase of $\beta$ powers, the brown for the low increases of $\beta$ powers, and the dark for the low reduction of $\beta$ powers. 

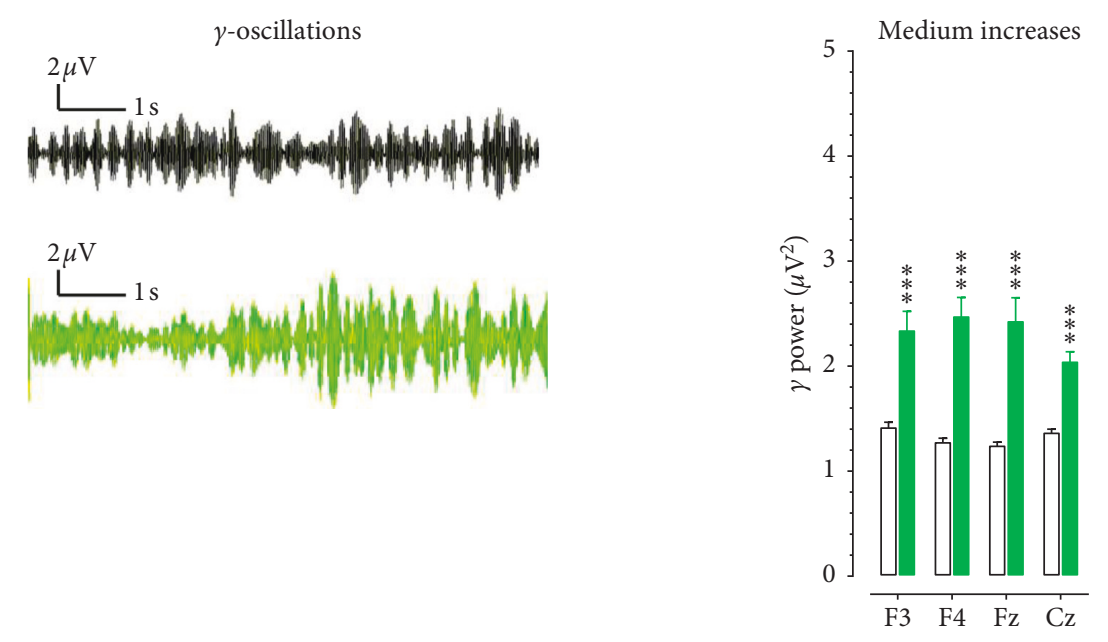

(a)

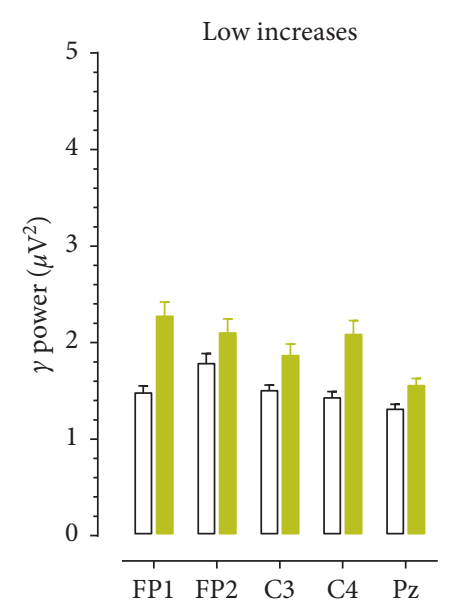

(c)

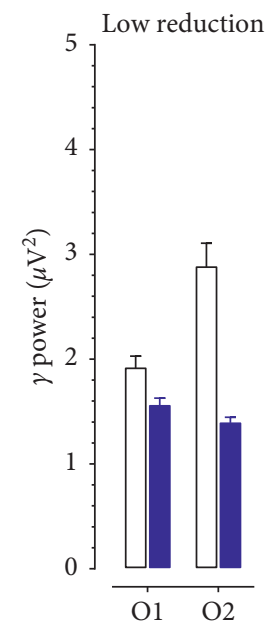

(e) (b)

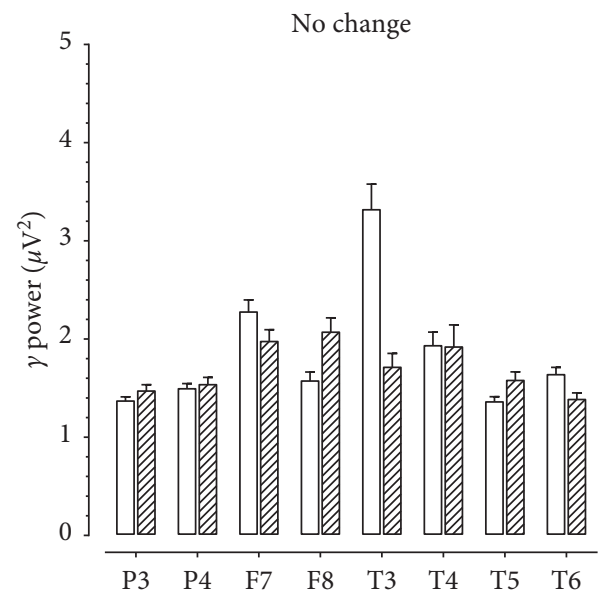

(d)

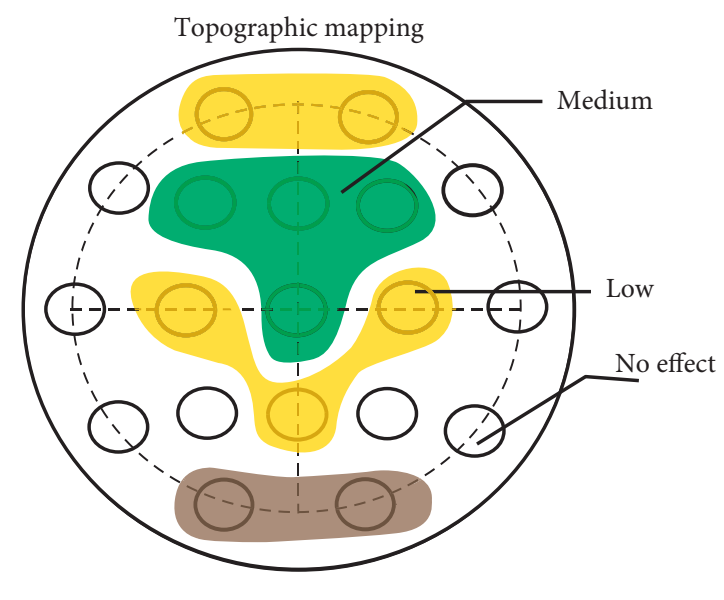

(f)

FIGURE 5: Topographic changes of $\gamma$ powers in patients with opioid use disorder. (a) Examples of EEG traces representing $10 \mathrm{~s} \gamma$ oscillations from a healthy control (top) and a patient with opioid use disorder. Horizontal scale bar, $1 \mathrm{~s}$; vertical scale bar, $2 \mu \mathrm{V}$. (b) The increase of $\gamma$ powers was 51-75\%, including F3, F4, Fz, and Cz. Open columns denote for the healthy control group, and solid green columns for the opioid group. NS, $P>0.05$. ${ }^{* * *} P<0.001$ vs. healthy controls determined by repeated measures ANOVA followed by post hoc Scheffe test. (c) The increase of $\beta$ powers was 11-50\% in FP1, FP2, C3, C4, F7, and F8. Open columns denote for the healthy control group, and solid brown columns for a low change in the opioid group. NS, $P>0.05$ vs. healthy control determined by repeated measures ANOVA. (d) The increase of $\beta$ powers was $<10 \%$ in P3, P4, T3, T4, T5, T6, and Pz. Open columns denote for the healthy control group, and zebra-striping columns for "no change" in the opioid group. NS, $P>0.05$ vs. healthy control determined by repeated measures ANOVA. (e) A tendency of reduction in $\beta$ powers was found in O1 and O2. Open columns denote for the healthy control group, and solid blue columns for a reduction of $\gamma$ oscillations in the opioid group. NS, $P>0.05$ vs. healthy control determined by repeated measures ANOVA. (f) Topographic correlation between $\gamma$ oscillation (30-50 Hz) and electrodes on the scalp. The green areas denote for a medium increase of $\gamma$ powers, the brown for the low $\gamma$ powers, and the dark area for a low reduction of $\gamma$ powers. 
Figure 5(c) shows a low increase of $\gamma$ powers in the FP1, FP2, C3, C4, and Pz electrodes. However, the effect was not significant $\left(F_{(1,30)}=1.287, P=0.266\right)$. Figure $5(d)$ shows a group of electrodes had no change in $\gamma$ powers, including P3, P4, F8, T3, T4, T5, and T6 $\left(F_{(1,30)}=0.150, P=0.702\right)$. Note that, although T3 powers were reduced by $48 \%$, it would be classified into "no effect" since T4 had less than $1 \%$ change compared with the electrode in the counterpart area. There was a tendency that $\mathrm{O} 1$ and $\mathrm{O} 2$ electrodes had a reduction in $\gamma$ powers (Figure 5(e)). However, this effect was not significant $\left(F_{(1,30)}=1.932, P=0.175\right)$. Figure 5 (f) displays a topographical response of $\gamma$ powers in opioid patients. The green area indicates medium increases of $\gamma$ powers, the brown for the low increases, and the dark represents the low reduction of $\gamma$ powers as measured at the scalp.

\subsection{Identifying Specificity of Electrodes That Could Be Used to Detect Changes in EEG Powers of Patients with Opioid Use Disorders}

3.3.1. Aberrant Distribution of Spectral Powers Across the Scalp in Patients with Opioid Use Disorder. In addition to the individual analysis, electrodes were grouped as the frontal (FP1, FP2, F3, F4, F7, F8, and Fz), temporal (T3, T4, T5, and T6), central (C3, C4, and Cz), parietal (P3, P4, and $\mathrm{Pz}$ ), and occipital electrodes (O1 and $\mathrm{O} 2)$. Figure 6 shows the mean powers in these areas of the patients compared with healthy controls. The $\delta$ powers were increased throughout all 5 areas. The $\theta$ powers were increased in 4 areas except for the occipitals. Although there was a reduction of $\alpha$ powers in sensorimotor regions (i.e., temporal, parietal, and occipital areas), this effect was not significant. The $\beta$ and $\gamma$ powers were increased mainly in the frontal and central areas, but not in temporal or parietal areas, and a nonsignificant reduction in occipitals.

\subsubsection{Venn Diagram Analysis to Identify Electrodes That} Could Detect Changes in Powers of Spectra as Many as Possible, including $\delta, \theta, \beta$, and $\gamma$ Oscillations. Individual electrodes which had significantly increased powers (based on results from Figures 1-6) are sorted into $\delta, \theta$, or $\beta / \gamma$ groups with a Venn diagram. As shown in Figure $7(\mathrm{a}), 15$ out of 19 electrodes are found in the $\delta$ group. In contrast, only 9 electrodes are found in the $\theta$ group. C3, C4, T4, F3, $\mathrm{F} 4, \mathrm{Fz}$, and $\mathrm{Cz}$ in the $\delta$ group are overlapped with $\theta$ groups. Both $\beta$ and $\gamma$ groups have F3, F4, Fz, and Cz. Overall, F3, F4, $\mathrm{Fz}$, and $\mathrm{Cz}$ are overlapped by $\delta, \theta, \beta / \gamma$ groups. Topographically, those 4 electrodes were next each other on the scalp (Figure 7(b)).

\section{Discussion}

The current study yielded several major findings. First, there was topographical distribution of $\delta, \theta, \alpha, \beta$, and $\gamma$ powers across the scalp of healthy controls in a closed-eye resting state. The $\delta$ powers were high in the frontal areas, while $\alpha$ and $\beta$ powers were dominant in the rear areas, particularly in the occipitals. However, the distribution pattern for $\theta$ and $\gamma$ powers was not apparent. Nevertheless, the data support the notion that spectral powers are characteristically distributed across the cortex, which can be considered as fundamental values in estimating mental health. Second, we found that changes in spectral powers were characteristic of patients with opioid use disorder, consistent with previous opioid research reports $[26,30]$. A majority of 19 electrodes could detect an increase of $\delta$ but not $\theta, \alpha, \beta$, or $\gamma$ powers. Interestingly, all except for the $\alpha$ component increased at F3, $\mathrm{F} 4, \mathrm{Fz}$, and $\mathrm{Cz}$. This suggests that these four electrodes may be more useful than others in assessing opioid use disorder. Third, we observed that $\alpha$ powers were reduced in the sensorimotor areas, particularly occipital $\mathrm{O} 1$ and $\mathrm{O} 2$ electrodes. Although the reduction was not statistically significant, it suggests that $\alpha$ oscillations in response to opioid use was different from other oscillations.

4.1. Topographic Analysis of Spectral Powers across the Scalp in Healthy Controls. In this study, EEG microstates exhibited two apparent patterns. First, EEG activity obtained from a given electrode was very similar to nearby or surrounding electrodes. As a result, changes in spectral powers appear gradual rather than sudden. For instance, $\mathrm{Cz}$ is located in the midline center surrounded by $\mathrm{C} 3, \mathrm{Fz}, \mathrm{C} 4$, and Pz. EEG activity on $\mathrm{Cz}$ was more or less similar to $\mathrm{C} 3, \mathrm{Fz}, \mathrm{C} 4$, or $\mathrm{Pz}$. This pattern could be applied to almost any other electrode, and thus changes in spectral powers of several nearby electrodes could be grouped for the data analysis. Second, the corresponding or counterpart electrodes located on two hemispheric sides (e.g., F7 vs. F8 and T5 vs. T6) had almost the same power levels and the same response to opioid use. One explanation for the similarity in power may be ascribed to the fact that there are the same or similar neural anatomies or microcircuits between two hemispheres, and thus the distribution of power levels appears to be symmetric as measured at the scalp. This also suggests that the apparatus used in the study had high quality in collecting EEG data.

However, there was a regional difference in power between frontal and rear areas, and between lobes (frontal, temporal, central, parietal, and occipital). The $\delta$ oscillations appear higher in the frontal area while $\alpha$ and $\beta$ are higher in the occipital area, consistent with previous observations in healthy controls [31-33]. The difference in spectral powers can be ascribed to the cortical microcircuits beneath electrodes. It has been observed that the dendritic fields of pyramidal neurons in the frontal cortex are several times greater than those in the occipital cortex [34]. In addition, deep brain projections to cortical layers may be different between cortical lobes. For instance, thalamic neurons provide greater inputs to the visual cortex while the hippocampal, ventral tegmental area (VTA), and many other nuclei predominately innervate the frontal cortex [35-37]. Thus, differences in the microcircuits would be the cause of differential powers of spectra received on the EEG electrodes, consistent with previous reports [38]. Taken together, levels of spectral powers may be characteristically distributed across scalps. 


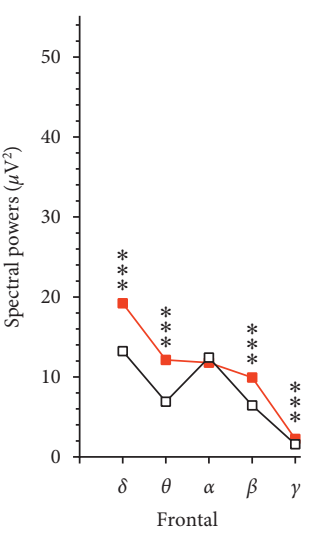

$$
\begin{aligned}
& \square \text { Healthy control } \\
& \square-\text { Opioid dependent }
\end{aligned}
$$

(a)

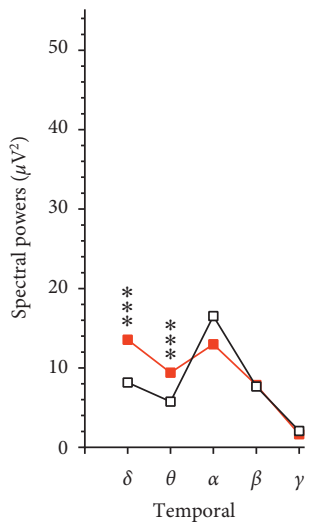

$\square$ Healthy control

(c)

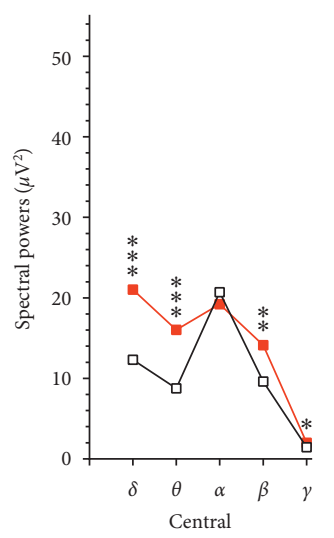

$\square-$ Healthy control

$\square$ Opioid dependent

(b)

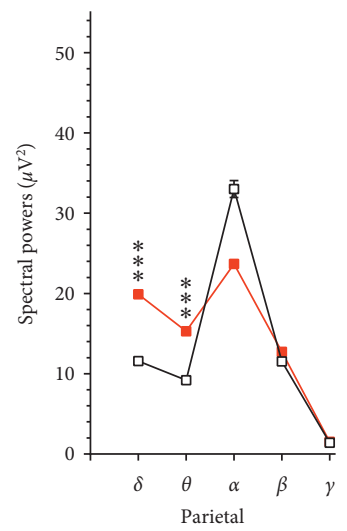

$\square-$ Healthy control

$\square$ Opioid dependent

(d)

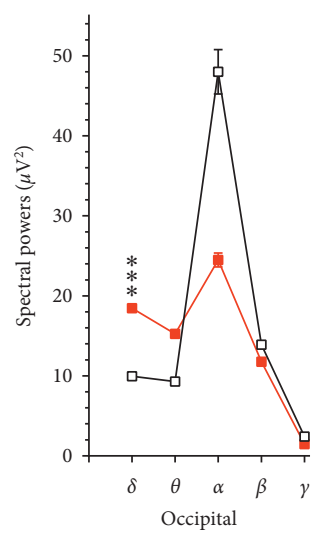

$\square$ Healthy control

$\square$ Opioid dependent

(e)

Figure 6: Comparative analysis of the 5 areas. (a) Compared to the respective frequency bands in health controls, there were increases in powers of $\delta, \theta, \beta$, and $\gamma$ oscillations, but not $\alpha$ oscillations of patients with opioid use disorder. ${ }^{* * *} P<0.001$ vs. health controls determined by unpaired Student's $t$-test. (b) Compared to the respective frequency bands in health controls, there were increases in powers of $\delta$ and $\theta$ oscillations, but not $\alpha, \beta$, or $\gamma$ oscillations of patients with opioid use disorder. ${ }^{* * *} P<0.001$ vs. health controls determined by unpaired Student's $t$-test. (c) Compared to the respective frequency bands in health controls, there were increases in powers of $\delta$, $\theta$, $\beta$, and $\gamma$ oscillations, but not $\alpha$ oscillations of patients with opioid use disorder. ${ }^{* * *} P<0.001$ vs. health controls determined by unpaired Student's $t$ test. (d) Compared to the respective frequency bands in health controls, there were increases in powers of $\delta$ and $\theta$ oscillations, but not $\alpha$, $\beta$, or $\gamma$ oscillations of patients with opioid use disorder. ${ }^{* * *} P<0.001$ vs. health controls determined by unpaired Student's $t$-test. (e) Compared to the respective frequency bands in health controls, there were increases in powers of $\delta$ oscillations, but not $\theta, \alpha, \beta$, or $\gamma$ oscillations of patients with opioid use disorder. ${ }^{* * *} P<0.001$ vs. health controls determined by unpaired Student's $t$-test. 


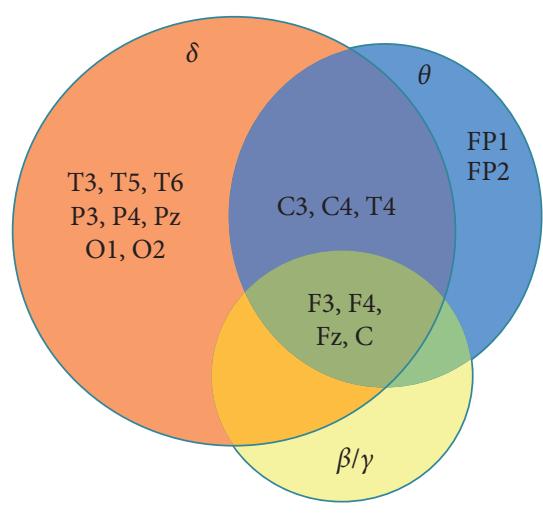

(a)

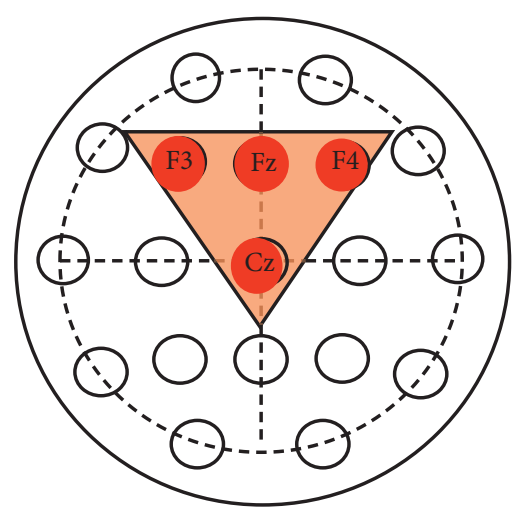

(b)

FIgURE 7: Venn diagram analysis to identify specificity of electrodes in which spectral powers were altered in patients with opioid use disorder. (a) The brown circle indicates $\delta$-specific electrodes, the blue circle for $\theta$-specific electrodes, and the yellow for $\beta / \gamma$-specific electrodes. (b) Topographic location of electrodes showing increases in $\delta, \theta, \beta$, and $\gamma$ powers.

TABLE 5: Classification of EEG spectral activity on scalps of patients with opioid use disorders. The activity was categorized into three classes as follows:

\begin{tabular}{|c|c|c|c|c|c|c|c|c|c|c|c|}
\hline \multirow{3}{*}{ Class 1} & \multirow{3}{*}{$\begin{array}{l}\delta \\
\theta\end{array}$} & \multicolumn{2}{|c|}{ Frontal } & \multicolumn{2}{|c|}{ Central } & \multicolumn{2}{|c|}{ Temporal } & \multicolumn{2}{|c|}{ Parietal } & \multicolumn{2}{|c|}{ Occipital } \\
\hline & & $\uparrow$ & $\checkmark$ & $\uparrow$ & $\checkmark$ & $\uparrow$ & $\checkmark$ & $\uparrow$ & $\checkmark$ & $\uparrow$ & $\checkmark$ \\
\hline & & $\uparrow$ & $\checkmark$ & $\uparrow$ & $\checkmark$ & $\uparrow$ & $\checkmark$ & $\uparrow$ & $\checkmark$ & $\uparrow$ & $x$ \\
\hline \multirow{2}{*}{ Class 2} & $\beta$ & $\uparrow$ & $\checkmark$ & $\uparrow$ & $\checkmark$ & $\leftrightarrow$ & $x$ & $\leftrightarrow$ & $x$ & $\downarrow$ & $x$ \\
\hline & $\gamma$ & $\uparrow$ & $\checkmark$ & $\uparrow$ & $\checkmark$ & $\leftrightarrow$ & $x$ & $\leftrightarrow$ & $x$ & $\downarrow$ & $x$ \\
\hline Class 3 & $\alpha$ & $\leftrightarrow$ & $x$ & $\leftrightarrow$ & $x$ & $\downarrow$ & $x$ & $\downarrow$ & $x$ & $\downarrow$ & $x$ \\
\hline
\end{tabular}

Note that frontal electrodes included FP1, FP2, F3, F4, F7, F8, and Fz; central electrodes included T3, T4, T5, and T6; temporal were C3, C4, and Cz; parietal were P3, P4, and Pz; and occipitals were $\mathrm{O} 1$ and $\mathrm{O} 2 . \uparrow \checkmark$, Increases in spectral powers with statistical significance. $\uparrow \boldsymbol{X}$, Increases in spectral powers but no statistical significance. $\leftrightarrow \boldsymbol{X}$, Neither increases nor decreases in spectral powers. $\downarrow \boldsymbol{X}$, Decreases in spectral powers with statistical significance.

4.2. Increases in $\delta, \theta, \beta$ and $\gamma$ Powers, but Not $\alpha$ Power, of Patients with Opioid Use Disorder. We found that, between 19 electrodes, there were 15 electrodes showing significant increases in $\delta$ oscillations, or $74 \%$ of total electrodes. In contrast, there were only $9(47 \%), 4(21 \%)$, and 4 electrodes $(21 \%)$ showing significant increases in $\theta, \beta$, and $\gamma$ powers, respectively. The findings are in line with some of the previous reports [39], but not others [30], demonstrating that $\delta$ oscillations are the major frequency band altered in patients with opioid use disorder. This suggests that $\delta$ oscillations are more reliable than other frequencies in estimating opioid use disorder. However, an individual electrode analysis could underestimate the importance of other powers (i.e., $\theta, \alpha, \beta$, or $\gamma$ ). For this reason, we also conducted group-oriented Venn diagram analysis highlighting significance in the frontal, central, temporal, parietal, and occipital areas. Results of our data analysis based on several approaches prompted us to suggest that changes of spectral powers in response to opioid use can be grouped into three subclasses. As illustrated in Table 5 , the $\delta$ and $\theta$ powers could be grouped together as class 1 , showing significant increases in almost all areas, suggesting that opioids likely modulate $\delta$ and $\theta$ activity across the cortex. The $\beta$ and $\gamma$ powers grouped as class 2 were mainly found in the frontal and central areas, implicating involvement of emotional control, not sensorimotor activity located in the temporal or parietal regions. Given that dopaminergic projections from the VTA to cortical areas mainly target the frontal regions [40], it would be of interest to explore whether dopaminergic activity altered by opioids is involved in changes of the $\beta$ and $\gamma$ powers. The $\alpha$ powers as class 3 could be exceptional and different from either class 1 or 2 , showing no increase on any region measured. Instead, there was a reduction of powers in the sensorimotor areas, such as temporal, parietal, and occipital regions (Figure 3 and Table 5). An advantage of such a categorization is that it allows the spectral powers to associate with specific brain areas. However, additional verification is needed to determine whether functional activity relevant to areas is indeed modulated or impaired in association with changes in spectral activity, which could be explored with event-related potential (ERP) measurements in patients with opioid abuse [26].

\subsection{Possible Mechanisms Responsible for Increases or De-} creases in Spectral Powers. The increases in powers can be interpreted as increasing synchronized activity of pyramidal neurons in the cortical microcircuits as measured by nearby electrodes, consistent with EEG theory [41]. This suggests that the inhibitory and excitatory balances in the microcircuits of neuronal activity have been modulated in the opioid patients. Mechanisms are not fully understood, but changes in neuroplasticity could be one of the causes that contribute to the modulation. Functional neuroplasticity refers to the enhancement of synaptic strength, involving IPSPs or EPSPs or both (see reviews [42]). While there is no 
direct effect of opioids on pyramidal neurons, the increasing synchronized effect is ascribed mainly to synaptic inputs from either GABAergic interneurons or deep brain afferents. Electrophysiological studies revealed that signal transduction pathways of GABAergic interneurons are impaired, and the spontaneous IPSPs are significantly increased in brain slices of chronic opioid rodents reported in some studies [43-45], but not others [46]. Thus, increasing EEG synchronization may be due to increasing GABAergic inputs to the pyramidal neurons. Consistent with the hypothesis, it was found that opioids no longer cause a reduction in IPSPs of brain slices obtained from chronic opioid-treated animals, but instead potentiate the IPSPs [44, 47, 48]. GABAergic activity is found to be increased in the cortical microcircuits, specifically the medial prefrontal cortex [49].

Glutamatergic pyramidal neurons in the cortex are the major carriers in which plasticity takes place [50]; also see reviews by [51]. Consistent with this, glutamate levels were increased in the brain of patients with opioid use disorder $[52,53]$. Signal transduction pathways, including NMDA- $\mathrm{Ca}^{2+}-\mathrm{NO}-\mathrm{cGMP}$ pathway, AC-cAMP-CREB pathway, and MAPK pathways are activated, which are critical for plasticity changes [54]. Involvement of glutamatergic neuroplasticity was also supported with observation in animal studies. For instance, the noncompetitive NMDA receptor blocker, MK-801 antagonized the changes of EEG spectral powers [55], and EPSP amplitudes of glutamatergic neurons synapsing to pyramidal neurons in the cortex were increased in rodents with chronic opioids [56]. In addition, chronic use of opioids sensitizes the expression of $D_{1}$ receptors on glutamatergic neurons in the basolateral amygdala that project to the cortex [56].

In addition to increases, we also observed reduction in spectral powers, mainly $\alpha$ oscillations in the sensorimotor areas measured with $\mathrm{O} 1$ and $\mathrm{O} 2$ electrodes. It is suggested that a reduction in power is indicative of desynchronization of neuronal activities associated with wakefulness, alertness, and intensive activity of the perceptional process in response to a task [57, 58]; also see an excellent review [59]. In contrast, increases in power may be attributed to synchronized IPSPs as patients become drowsy during cortical idling or active inhibition [60]. Increases in power are also associated with synchronized EPSPs for hallucinations [61], and abuse of psychostimulants $[62,63]$. Despite this, the exact mechanism underlying relationship between changes in spectral powers and mental health is still not understood.

\section{Conclusions}

Consistent with previous reports [23, 26, 39, 64], we demonstrate that changes in spectral rhythms took place in some, but not all 19 electrodes of patients with opioid use disorder. Major changes were found at F3, F4, Fz, and Cz. Considering that EEG rhythms are electrical signals reflecting activity of neural circuits consisting of glutamatergic, GABAergic, and many other types of neurons, the findings of the present study are limited by the study design focusing on opioid cases only, and unlikely applicable to polysubstance users. This is because the detrimental effect of opioids is ascribed to mainly GABAergic dysfunction $[18,19]$, other types of neurons contributed to changes in EEG rhythms in polysubstance use was not examined in the present study. We found that patients with methamphetamine or alcohol use disorder were shown different changes in EEG rhythms (unpublished observation), supporting this hypothesis. Furthermore, the present investigation included only 16 matched cases. Strict validation with large sample sizes is needed before being generalized to all opioid users. It is worthy to note that EEG data were recorded while subjects were asked to have eyes closed. It would be interesting in the future study to investigate event-related potentials (ERP) in patients with opioid use disorders $[26,65]$.

\section{Data Availability}

The authors declare that data supporting the findings of this study are available within the article.

\section{Ethical Approval}

This research was approved by the Institutional Review Board (IRB) from Florida Atlantic University (\#1223155) and Ross University School of Veterinary Medicine.

\section{Conflicts of Interest}

The authors declare that they have no conflicts of interest.

\section{Acknowledgments}

The authors would like to thank FHE Health for granting permission to utilize their data for this study. We would like to extend our gratitude to the dedicated staff at the Neurorehabilitation department at FHE for collecting the data and helping us to make our work as smooth as possible. Additionally, the authors thank Dr. Ximena Levy for her knowledge over human research review and Jeffrey Clark for his excellent IT support, both of them are staff at Florida Atlantic University.

\section{References}

[1] R. Ahrnsbrak, J. Bose, S. L. Hedden, R. N. Lipari, E. Park-Lee, and P. Tice, "Key substance use and mental health indicators in the United States: results from the 2016 National survey on drug use and health," Substance Abuse and Mental Health Services Administration, HHS Publication, Center for Behavioral Health Statistics and Quality, Rockville, MD, USA, 2017.

[2] NIH, "Overdose death rates," 2017, https://www.drugabuse. gov/related-topics/trends-statistics/overdose-death-rates.

[3] A. M. Dela Cruz, "Diagnosis and treatment of prescription opioid use disorder in patients with chronic noncancer pain," The American Journal of Psychiatry, vol. 9, pp. 2-5, 2015.

[4] J. Prosser, E. D. London, and I. I. Galynker, "Sustained attention in patients receiving and abstinent following methadone maintenance treatment for opiate dependence: performance and neuroimaging results," Drug and Alcohol Dependence, vol. 104, no. 3, pp. 228-240, 2009. 
[5] B. J. Roach, D. C. D'Souza, J. M. Ford, and D. H. Mathalon, "Test-retest reliability of time-frequency measures of auditory steady-state responses in patients with schizophrenia and healthy controls," NeuroImage: Clinical, vol. 23, Article ID 101878, 2019.

[6] A. Vogrig, B. Joubert, N. André-Obadia, G. L. Gigli, S. Rheims, and J. Honnorat, "Seizure specificities in patients with antibody-mediated autoimmune encephalitis," Epilepsia, vol. 60, no. 8, pp. 1508-1525, 2019.

[7] R. E. Rosch, S. Wright, G. Cooray et al., "NMDA-receptor antibodies alter cortical microcircuit dynamics," Proceedings of the National Academy of Sciences, vol. 115, no. 42, pp. E9916-E9925, 2018.

[8] M. X. Cohen, "Where does EEG come from and what does it mean?," Trends in Neurosciences, vol. 40, no. 4, pp. 208-218, 2017.

[9] K. Taki, T. Kaneko, and N. Mizuno, "A group of cortical interneurons expressing $\mu$-opioid receptor-like immunoreactivity: a double immunofluorescence study in the rat cerebral cortex," Neuroscience, vol. 98, no. 2, pp. 221-231, 2000.

[10] F.-Q. Huo, J. Wang, Y.-Q. Li, T. Chen, F. Han, and J.-S. Tang, "GABAergic neurons express $\mu$-opioid receptors in the ventrolateral orbital cortex of the rat," Neuroscience Letters, vol. 382, no. 3, pp. 265-268, 2005.

[11] K. R. Svoboda and C. R. Lupica, "Opioid inhibition of hippocampal interneurons via modulation of potassium and hyperpolarization-activated cation (Ih) currents," The Journal of Neuroscience, vol. 18, no. 18, pp. 7084-7098, 1998.

[12] E. Yokota, Y. Koyanagi, K. Yamamoto, Y. Oi, N. Koshikawa, and M. Kobayashi, "Opioid subtype- and cell-type-dependent regulation of inhibitory synaptic transmission in the rat insular cortex," Neuroscience, vol. 339, pp. 478-490, 2016.

[13] J. Louvel, C. Papatheodoropoulos, A. Siniscalchi et al., "GABA-mediated synchronization in the human neocortex: elevations in extracellular potassium and presynaptic mechanisms," Neuroscience, vol. 105, no. 4, pp. 803-813, 2001.

[14] A. L. Svingos, M. Garzón, E. E. O. Colago, and V. M. Pickel, " $\mu$-Opioid receptors in the ventral tegmental area are targeted to presynaptically and directly modulate mesocortical projection neurons," Synapse, vol. 41, no. 3, pp. 221-229, 2001.

[15] C. Graversen, A. M. Drewes, and D. Farina, "Support vector machine classification of multi-channel EEG traces: a new tool to analyze the brain response to morphine treatment," in Proceedings of the Annual International Conference of the IEEE Engineering in Medicine and Biology, pp. 992-995, Buenos Aires, Argentina, August 2010.

[16] G. Montandon, S. L. Cushing, F. Campbell, E. J. Propst, R. L. Horner, and I. Narang, "Distinct cortical signatures associated with sedation and respiratory rate depression by morphine in a pediatric population," Anesthesiology, vol. 125, no. 5, pp. 889-903, 2016.

[17] N. Sun, Y. Li, S. Tian et al., "Dynamic changes in orbitofrontal neuronal activity in rats during opiate administration and withdrawal," Neuroscience, vol. 138, no. 1, pp. 77-82, 2006.

[18] X. Chen, H. G. Marrero, R. Murphy, Y.-J. Lin, and J. E. Freedman, "Altered gating of opiate receptor-modulated $\mathrm{K}^{+}$channels on amygdala neurons of morphine-dependent rats," Proceedings of the National Academy of Sciences, vol. 97, no. 26, pp. 14692-14696, 2000.

[19] F. J. Meye, R. van Zessen, M. P. Smidt, R. A. H. Adan, and G. M. J. Ramakers, "Morphine withdrawal enhances constitutive -opioid receptor activity in the ventral tegmental area," Journal of Neuroscience, vol. 32, no. 46, pp. 16120-16128, 2012.
[20] S. M. Assadi, R. Radgoodarzi, and S. A. Ahmadi-Abhari, "Baclofen for maintenance treatment of opioid dependence: a randomized double-blind placebo-controlled clinical trial [ISRCTN32121581]," BMC Psychiatry, vol. 3, no. 1, p. 16, 2003.

[21] M. A. Davis, L. A. Lin, H. Liu, and B. D. Sites, "Prescription opioid use among adults with mental health disorders in the United States," The Journal of the American Board of Family Medicine, vol. 30, no. 4, pp. 407-417, 2017.

[22] P. Olejniczak, "Neurophysiologic basis of EEG," Journal of Clinical Neurophysiology, vol. 23, no. 3, pp. 186-189, 2006.

[23] A. A. Fingelkurts, A. A. Fingelkurts, R. Kivisaari et al., "Reorganization of the composition of brain oscillations and their temporal characteristics in opioid dependent patients," Progress in Neuro-Psychopharmacology and Biological Psychiatry, vol. 30, no. 8, pp. 1453-1465, 2006.

[24] H. F. Ieong and Z. Yuan, "Resting-State neuroimaging and neuropsychological findings in opioid use disorder during abstinence: a review," Frontiers in Human Neuroscience, vol. 11, p. 169, 2017.

[25] G. Y. Wang, R. Kydd, T. A. Wouldes, M. Jensen, and B. R. Russell, "Changes in resting EEG following methadone treatment in opiate addicts," Clinical Neurophysiology, vol. 126, no. 5, pp. 943-950, 2015.

[26] F. Motlagh, F. Ibrahim, R. Rashid, N. Shafiabady, T. Seghatoleslam, and H. Habil, "Acute effects of methadone on EEG power spectrum and event-related potentials among heroin dependents," Psychopharmacology, vol. 235, no. 11, pp. 3273-3288, 2018.

[27] Z. Ma, M. Rudacille, H. M. Prentice, and R. Tao, "Characterization of electroencephalographic and biochemical responses at 5-HT promoting drug-induced onset of serotonin syndrome in rats," Journal of Neurochemistry, vol. 125, no. 5, pp. 774-789, 2013.

[28] I. M. Shokry, V. Sinha, G. Da Silva, S. B. Park, J. J. Callanan, and R. Tao, "Comparison of electroencephalogram (EEG) response to MDPV versus the hallucinogenic drugs MK-801 and ketamine in rats," Experimental Neurology, vol. 313, pp. 26-36, 2018.

[29] R. W. Thatcher, "Validity and reliability of quantitative electroencephalography," Journal of Neurotherapy, vol. 14, no. 2, pp. 122-152, 2010.

[30] A. A. Fingelkurts, S. Kähkönen, A. A. Fingelkurts et al., "Composition of EEG oscillations and their temporal characteristics: methadone treatment," International Journal of Psychophysiology, vol. 64, no. 2, pp. 130-140, 2007.

[31] M. Munch, V. Knoblauch, K. Blatter et al., "The frontal predominance in human EEG delta activity after sleep loss decreases with age," European Journal of Neuroscience, vol. 20, no. 5, pp. 1402-1410, 2004.

[32] M. Toscani, T. Marzi, S. Righi, M. P. Viggiano, and S. Baldassi, "Alpha waves: a neural signature of visual suppression," Experimental Brain Research, vol. 207, no. 3-4, pp. 213-219, 2010.

[33] B. Claudio, D. P. Claudio, B. Marina et al., "Occipital sources of resting state alpha rhythms are related to local gray matter density in subjects with amnesic mild cognitive impairment and alzheimers disease," Neurobiology of Aging, vol. 36, no. 2, pp. 556-570, 2015.

[34] G. N. Elston, "Cortex, cognition and the cell: new insights into the pyramidal neuron and prefrontal function," Cerebral Cortex, vol. 13, no. 11, pp. 1124-1138, 2003.

[35] B. P. Godsil, J. P. Kiss, M. Spedding, and T. M. Jay, "The hippocampal-prefrontal pathway: the weak link in psychiatric 
disorders?," European Neuropsychopharmacology, vol. 23, no. 10, pp. 1165-1181, 2013.

[36] W. Sun, Z. Tan, B. D. Mensh, and N. Ji, "Thalamus provides layer 4 of primary visual cortex with orientation- and direction-tuned inputs," Nature Neuroscience, vol. 19, no. 2, pp. 308-315, 2016.

[37] W. C. Buchta, S. V. Mahler, B. Harlan, G. S. Aston-Jones, and A. C. Riegel, "Dopamine terminals from the ventral tegmental area gate intrinsic inhibition in the prefrontal cortex," Physiological Reports, vol. 5, no. 6, Article ID e13198, 2017.

[38] G. Buzsáki and X.-J. Wang, "Mechanisms of gamma oscillations," Annual Review of Neuroscience, vol. 35, no. 1, pp. 203-225, 2012.

[39] M. K. Greenwald and T. A. Roehrs, "Mu-opioid self-administration vs passive administration in heroin abusers produces differential EEG activation," Neuropsychopharmacology, vol. 30, no. 1, pp. 212-221, 2005.

[40] B. L. Lewis and P. O'Donnell, "Ventral tegmental area afferents to the prefrontal cortex maintain membrane potential "up" states in pyramidal neurons via D1 dopamine receptors," Cerebral Cortex, vol. 10, no. 12, pp. 1168-1175, 2000.

[41] E. Lowet, M. J. Roberts, P. Bonizzi, J. Karel, and P. De Weerd, "Quantifying neural oscillatory synchronization: a comparison between spectral coherence and phase-locking value approaches," PloS One, vol. 11, Article ID e0146443, , 2016.

[42] J.-M. Fritschy and P. Panzanelli, "GABA ${ }_{\mathrm{A}}$ receptors and plasticity of inhibitory neurotransmission in the central nervous system," European Journal of Neuroscience, vol. 39, no. 11, pp. 1845-1865, 2014.

[43] A. Bonci and J. T. Williams, "Increased probability of GABA release during withdrawal from morphine," The Journal of Neuroscience, vol. 17, no. 2, pp. 796-803, 1997.

[44] T. Jolas, E. J. Nestler, and G. K. Aghajanian, "Chronic morphine increases GABA tone on serotonergic neurons of the dorsal raphe nucleus: association with an up-regulation of the cyclic AMP pathway," Neuroscience, vol. 95, no. 2, pp. 433-443, 1999.

[45] L. Wang, M. Shen, C. Jiang, L. Ma, and F. Wang, "Parvalbumin interneurons of central amygdala regulate the negative affective states and the expression of corticotrophin-releasing hormone during morphine withdrawal," The International Journal of Neuropsychopharmacology, vol. 19, no. 11, Article ID pyw060, 2016.

[46] A. R. Wilson-Poe, H.-J. Jeong, and C. W. Vaughan, "Chronic morphine reduces the readily releasable pool of GABA, a presynaptic mechanism of opioid tolerance," The Journal of Physiology, vol. 595, no. 20, pp. 6541-6555, 2017.

[47] A. Madhavan, A. Bonci, and J. L. Whistler, "Opioid-Induced GABA potentiation after chronic morphine attenuates the rewarding effects of opioids in the ventral tegmental area," Journal of Neuroscience, vol. 30, no. 42, pp. 14029-14035, 2010.

[48] M. Bajo, S. G. Madamba, M. Roberto, and G. R. Siggins, "Acute morphine alters GABAergic transmission in the central amygdala during naloxone-precipitated morphine withdrawal: role of cyclic AMP," Frontiers in Integrative Neuroscience, vol. 8, p. 45, 2014.

[49] M. C. Van den Oever, B. R. Lubbers, N. A. Goriounova et al., "Extracellular matrix plasticity and GABAergic inhibition of prefrontal cortex pyramidal cells facilitates relapse to heroin seeking," Neuropsychopharmacology, vol. 35, no. 10, pp. 2120-2133, 2010.

[50] M. C. Van den Oever, N. A. Goriounova, K. Wan Li et al., "Prefrontal cortex AMPA receptor plasticity is crucial for cue- induced relapse to heroin-seeking," Nature Neuroscience, vol. 11, no. 9, pp. 1053-1058, 2008.

[51] M. Hearing, "Prefrontal-accumbens opioid plasticity: implications for relapse and dependence," Pharmacological Research, vol. 139, pp. 158-165, 2019.

[52] M. K. Greenwald, E. A. Woodcock, D. Khatib, and J. A. Stanley, "Methadone maintenance dose modulates anterior cingulate glutamate levels in heroin-dependent individuals: a preliminary in vivo ${ }^{1} \mathrm{H}$ MRS study," Psychiatry Research: Neuroimaging, vol. 233, no. 2, pp. 218-224, 2015.

[53] X. L. Liu, L. Li, J. N. Li et al., "Quantifying absolute glutamate concentrations in nucleus accumbens of prescription opioid addicts by using ${ }^{1} \mathrm{H}$ MRS," Brain and Behavior, vol. 7, no. 8, Article ID e00769, 2017.

[54] Y. Guo, H.-L. Wang, X.-H. Xiang, and Y. Zhao, "The role of glutamate and its receptors in mesocorticolimbic dopaminergic regions in opioid addiction," Neuroscience \& Biobehavioral Reviews, vol. 33, no. 6, pp. 864-873, 2009.

[55] K. A. Haberny and G. A. Young, "Interactive effects of MK801 and morphine on EEG, EEG power spectra and behavior in rats: II. Morphine dependence," European Journal of Pharmacology, vol. 261, pp. 11-16, 1994.

[56] J. Song, M. Chen, Y. Dong, B. Lai, and P. Zheng, "Chronic morphine selectively sensitizes the effect of D1 receptor agonist on presynaptic glutamate release in basolateral amygdala neurons that project to prelimbic cortex," Neuropharmacology, vol. 133, pp. 375-384, 2018.

[57] T. Hartmann, W. Schlee, and N. Weisz, "It's only in your head: expectancy of aversive auditory stimulation modulates stimulus-induced auditory cortical alpha desynchronization," NeuroImage, vol. 60, no. 1, pp. 170-178, 2012.

[58] S. M. Haigh, N. R. Cooper, and A. J. Wilkins, "Chromaticity separation and the alpha response," Neuropsychologia, vol. 108, pp. 1-5, 2018.

[59] W. Klimesch, "Alpha-band oscillations, attention, and controlled access to stored information," Trends in Cognitive Sciences, vol. 16, no. 12, pp. 606-617, 2012.

[60] P. Sauseng, W. Klimesch, M. Doppelmayr, T. Pecherstorfer, R. Freunberger, and S. Hanslmayr, "EEG alpha synchronization and functional coupling during top-down processing in a working memory task," Human Brain Mapping, vol. 26, no. 2, pp. 148-155, 2005.

[61] E. Angelopoulos, E. Koutsoukos, A. Maillis, G. N. Papadimitriou, and C. Stefanis, "Cortical interactions during the experience of auditory verbal hallucinations," The Journal of Neuropsychiatry and Clinical Neurosciences, vol. 23, no. 3, pp. 287-293, 2011.

[62] T. F. Newton, I. A. Cook, A. D. Kalechstein et al., "Quantitative EEG abnormalities in recently abstinent methamphetamine dependent individuals," Clinical Neurophysiology, vol. 114, no. 3, pp. 410-415, 2003.

[63] M. S. Reid, F. Flammino, B. Howard, D. Nilsen, and L. S. Prichep, "Topographic imaging of quantitative EEG in response to smoked cocaine self-administration in humans," Neuropsychopharmacology, vol. 31, no. 4, pp. 872-884, 2006.

[64] A. G. Polunina and D. M. Davydov, "EEG spectral power and mean frequencies in early heroin abstinence," Progress in Neuro-Psychopharmacology and Biological Psychiatry, vol. 28, no. 1, pp. 73-82, 2004.

[65] B. Su, S. Wang, A. Sumich et al., "Reduction in N2 amplitude in response to deviant drug-related stimuli during a twochoice oddball task in long-term heroin abstainers," Psychopharmacology, vol. 234, no. 21, pp. 3195-3205, 2017. 


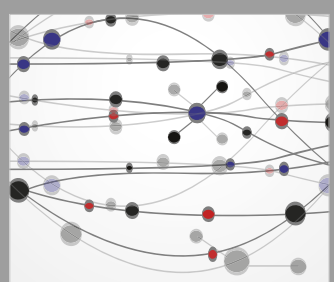

The Scientific World Journal
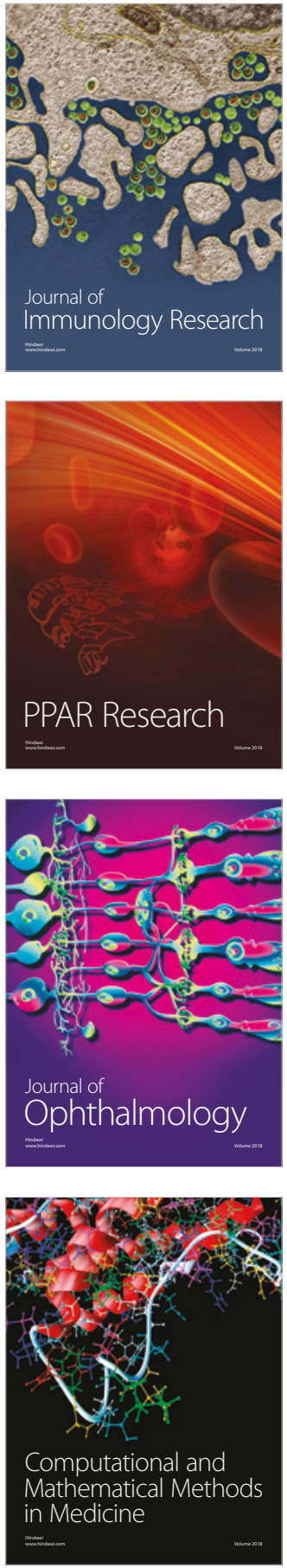

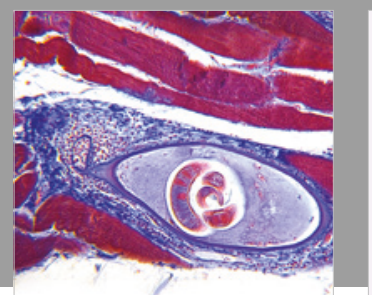

Gastroenterology Research and Practice

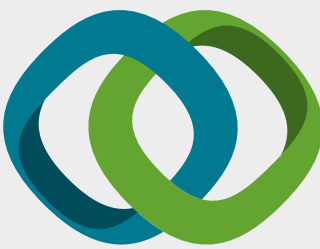

\section{Hindawi}

Submit your manuscripts at

www.hindawi.com
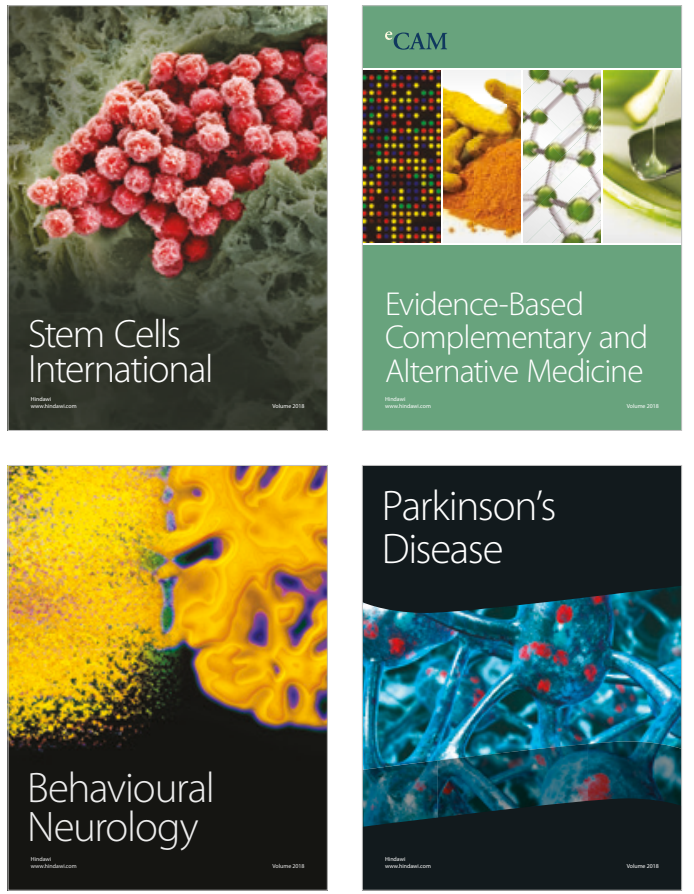

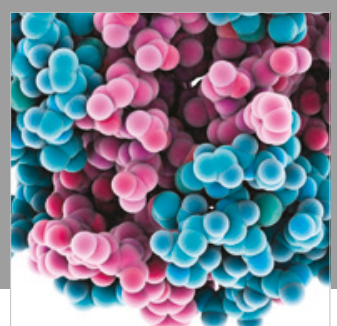

ournal of

Diabetes Research

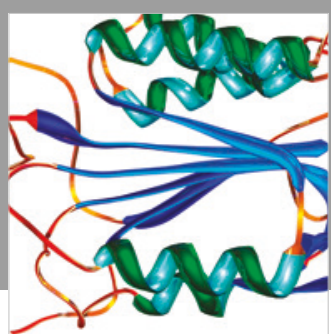

Disease Markers
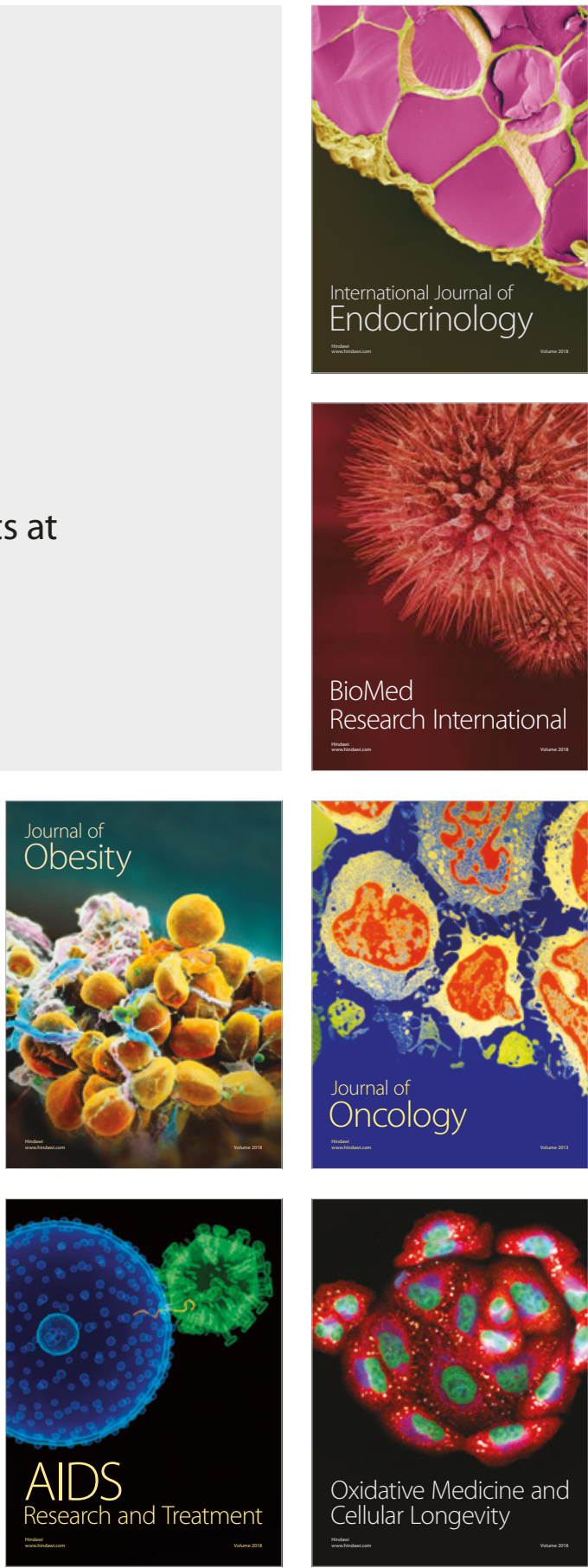